\title{
Identifying candidate genes for 2p15p16.1 microdeletion syndrome using clinical, genomic, and functional analysis
}

\author{
Hani Bagheri, ${ }^{1,2}$ Chansonette Badduke, ${ }^{1,2}$ Ying Qiao, ${ }^{1,2}$ Rita Colnaghi, ${ }^{3}$ Iga Abramowicz, ${ }^{3}$ \\ Diana Alcantara, ${ }^{3}$ Christopher Dunham, ${ }^{1}$ Jiadi Wen, ${ }^{4}$ Robert S. Wildin, ${ }^{5}$ Malgorzata J.M. Nowaczyk, ${ }^{6}$ \\ Jennifer Eichmeyer, ${ }^{7}$ Anna Lehman, ${ }^{8}$ Bruno Maranda, ${ }^{9}$ Sally Martell,, ${ }^{1,2}$ Xianghong Shan, ${ }^{10}$ \\ Suzanne M.E. Lewis, ${ }^{8}$ Mark O'Driscoll, ${ }^{3}$ Cheryl Y. Gregory-Evans, ${ }^{10}$ and Evica Rajcan-Separovic ${ }^{1,2}$ \\ 'Department of Pathology and Laboratory Medicine, University of British Columbia (UBC), Vancouver, British Columbia, \\ Canada. ${ }^{2}$ Child and Family Research Institute, Vancouver, British Columbia, Canada. ${ }^{3} H u m a n$ DNA Damage Response \\ Disorders Group, Genome Damage and Stability Centre, University of Sussex, Brighton, United Kingdom. \\ ${ }^{4}$ University of Texas at Dallas, Dallas, Texas, USA. ${ }^{5}$ St. Luke's Children's Hospital, Boise, Idaho, USA. \\ ${ }^{6}$ Department of Pathology and Molecular Medicine, McMaster University Medical Centre, Hamilton, \\ Ontario, Canada. ${ }^{7}$ St. Luke's Mountain States Tumor Institute, Boise, Idaho, USA. ${ }^{8}$ Department of Medical Genetics, \\ UBC, Vancouver, British Columbia, Canada. ${ }^{9}$ Medical Genetics, Université de Sherbrooke, Sherbrooke, Québec, Canada. \\ ${ }^{10}$ Department of Ophthalmology, UBC, Vancouver, British Columbia, Canada.
}

The 2p15p16.1 microdeletion syndrome has a core phenotype consisting of intellectual disability, microcephaly, hypotonia, delayed growth, common craniofacial features, and digital anomalies. So far, more than 20 cases of 2p15p16.1 microdeletion syndrome have been reported in the literature; however, the size of the deletions and their breakpoints vary, making it difficult to identify the candidate genes. Recent reports pointed to 4 genes (XP01, USP34, BCL11A, and REL) that were included, alone or in combination, in the smallest deletions causing the syndrome. Here, we describe 8 new patients with the 2p15p16.1 deletion and review all published cases to date. We demonstrate functional deficits for the above 4 candidate genes using patients' lymphoblast cell lines (LCLs) and knockdown of their orthologs in zebrafish. All genes were dosage sensitive on the basis of reduced protein expression in LCLs. In addition, deletion of XPO1, a nuclear exporter, cosegregated with nuclear accumulation of one of its cargo molecules (rpS5) in patients' LCLs. Other pathways associated with these genes (e.g., NF-кB and Wnt signaling as well as the DNA damage response) were not impaired in patients' LCLs. Knockdown of xpo1a, rel, bcl11aa, and bcl11ab resulted in abnormal zebrafish embryonic development including microcephaly, dysmorphic body, hindered growth, and small fins as well as structural brain abnormalities. Our multifaceted analysis strongly implicates XP01, REL, and BCL11A as candidate genes for 2p15p16.1 microdeletion syndrome.

Authorship note: $\mathrm{H}$. Bagheri and C. Badduke contributed equally to this work.

Conflict of interest: The authors have declared that no conflict of interest exists.

Submitted: November 30, 2015 Accepted: February 9, 2016 Published: March 17, 2016

Reference information: JCI Insight. 2016;1(3):e85461. doi:10.1172/jci.insight.85461.

\section{Introduction}

The 2p15p16.1 microdeletion syndrome (Online Mendelian Inheritance in Man [OMIM] 612513) is a recently recognized genomic disorder that we first described in 2 phenotypically similar individuals with idiopathic intellectual disability (ID) studied by chromosomal microarrays (CMAs) (1). Since the initial report, detailed clinical phenotypes for 25 subjects have been reported, demonstrating common phenotypic abnormalities including delayed neurocognitive development, microcephaly, consistent and recognizable facial dysmorphism (telecanthus, broad and high nasal root, ptosis, long and smooth philtrum), feeding problems, a variety of head shape and structural brain abnormalities, hypotonia, and digital anomalies (reviewed previously in refs. 2-6).

The deletions reported in the $2 \mathrm{p} 15 \mathrm{p} 16.1$ region are highly variable in size $(0.1-8 \mathrm{Mb})$, and the existence of nonoverlapping copy number variants (CNVs) within the $2 \mathrm{p} 15 \mathrm{p} 16.1$ deletion region complicate efforts to delineate a common critical region for the syndrome. In addition, some studies suggested that 

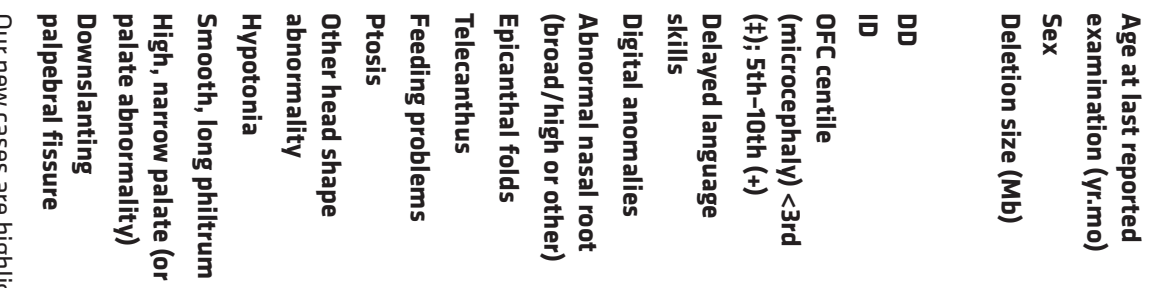

$1++++1+++++++6.74613+\quad$ Florisson et al., 2013 (1)

3 vo

일

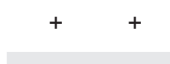

$++6.7461 \leq 0$
$+++7.8918 \leq 0$

Rajcan-Separovic et al., 2007 (2)

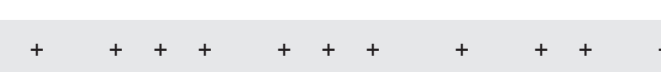

$++9.5744 \leq$

$3 \quad \rightarrow$

Case 1

$\frac{\overrightarrow{\mathrm{D}}}{\mathrm{D}}$

$+++3.5278 \pi \quad 6$ Prontera et al., 2011

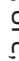

贾

$++6.1122 \pi \infty$

Rajcan-Separovic et al., 2007 (1)

䆑

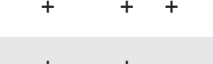

$+\quad+2.01263 \underset{\infty}{\infty}$ Case 2

$++++++++++++3.45123 \underset{N}{\mathrm{~N}}$

de Leeuw et al., 2008

$+++6.6777 \pi \vec{\omega}$

Florisson et al., 2013 (2)

$++5.36243 w$ Case 3

$++3.3497 \pi \rightarrow$ Felix et al., 2010

家
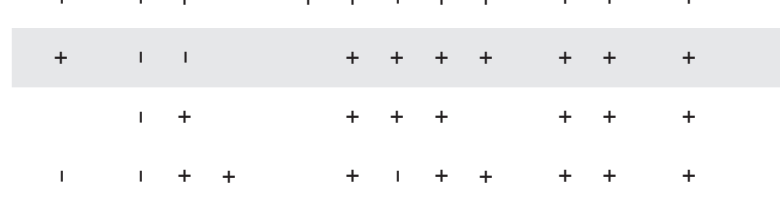

$+++3.1441 \pi \quad+\quad \dot{\sigma} \quad$ Liang et al., 2009

।。 $0.8758 \pi \omega$ Balci et al., 2015

$+++1.0295$

or Basak et al., 2015 (2)

$+\quad+6.3100 \leq \vec{i} \quad$ Jorgez et al., 2014 (4)

富

$+++1.6818 \pi \quad \rightarrow \quad$ Ottolini et al., 2015

薄.

$+++2.5050 \leq \stackrel{\circ}{v}$

Piccione et al., 2012 (2)

$+++0.6430 \pi+$

Piccione et al., 2012 (1)

害

$1++0.9710 \pi \overrightarrow{\vec{\sigma}}$ Case 4

$+++2.4724 \pi \sim$ Hucthagowder et al., 2012

콩.

旁

离

离

穿.

总

宽 融

资

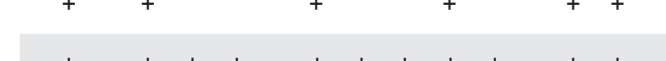

$++0.2030 \leq \quad \Rightarrow \quad$ Peter et al., 2014

$+++0.4380 \pi \quad \vec{\perp} \quad$ Hancarova et al., 2013

$+\quad+4.60003$ б

Jorgez et al., 2014 (5)

$++4.5927$

3 ने

$++2.3900$

Case 5

।。+ + $0.5833 \leq$

Jorgez et al., 2014 (3)

苟

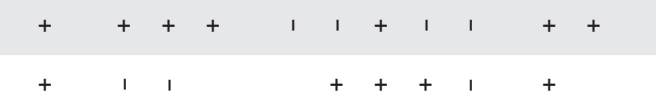

$+++0.3595 \leq$

Chabchoub et al., 2008

党足

竞

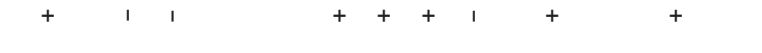

$++0.2378 \leq w$

Case 6

$+++0.2327 \leq \simeq$ Fannemel et al., 2014

'。 $\quad+2.7500 \leq \vec{\perp} \quad$ Jorgez et al., 2014 (6)

\#高.

$++2.6672 \leq \vec{N} \quad$ Case 7

言 营

$+++3.5240 \pi \quad \vec{\infty}$ Shimojima et al., 2015 (2)

$\#++0.10293 \vec{\Phi}$

Ronzoni et al., 2015

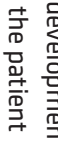

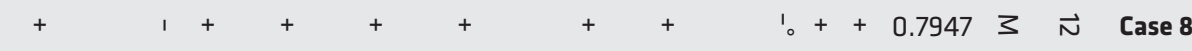

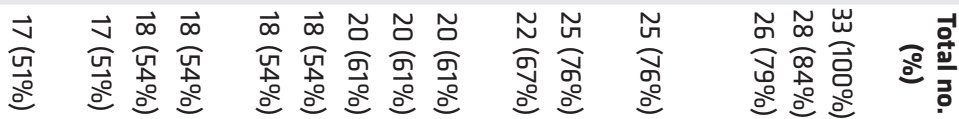


Case 2
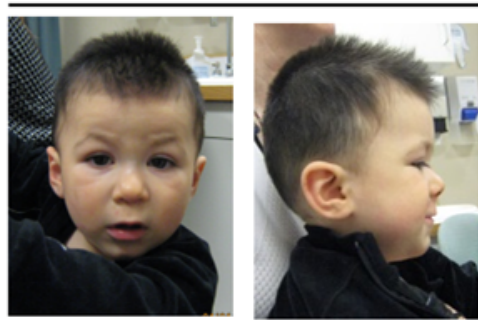

Case 3

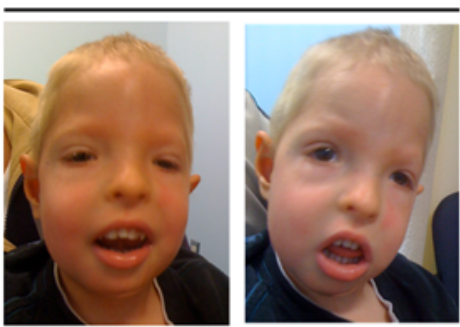

Case 4

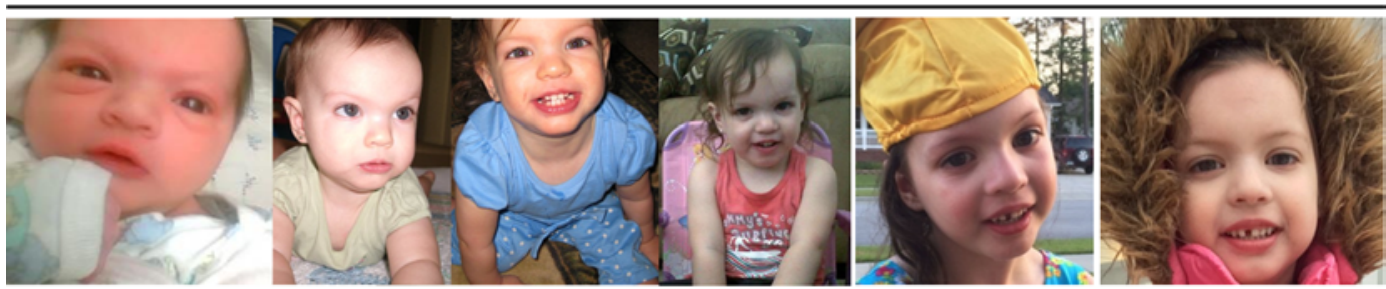

Case 7

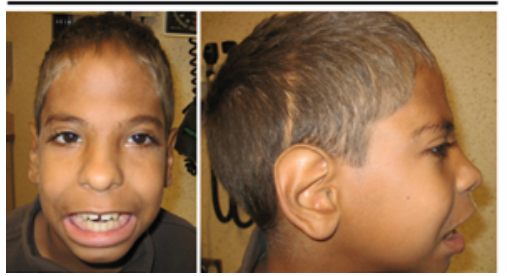

Case 8
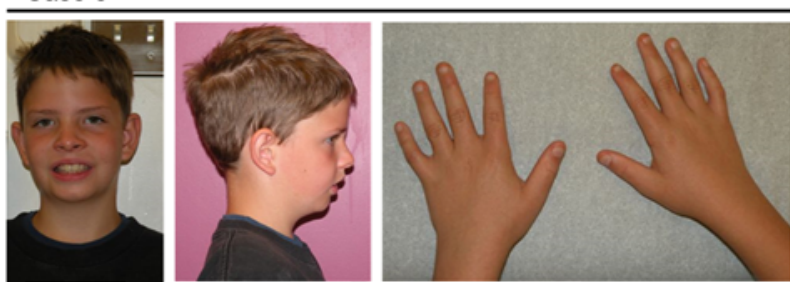

Figure 1. Physical features of the 2p15p16.1 microdeletion carriers. Images of the patients in cases 2, 3, 4, 7, and 8 illustrating the craniofacial and digital abnormalities seen in 2p15p16.1 microdeletion syndrome. All patients had microcephaly and/or head shape abnormalities (except case 4), and at least 1 unique facial feature including hypertelorism, epicanthal folds, telecanthus, short/downslanting palpebral fissure, ptosis, and abnormal nasal root. Digital anomalies were also a common feature in these patients.

regulatory elements (enhancers) in the region could also play a role (7). Nevertheless, several deletions involving individual genes or smaller numbers of genes were reported $(2-5,8-10)$ in patients with phenotypes in keeping with 2p15p16.1 microdeletion syndrome. Among these, 4 genes (XPO1 [Mendelian Inheritance in Man (MIM) 602559], BCL11A [MIM 606557], REL [MIM 164910], and USP34 [MIM $615295]$ ) were found to be repeatedly involved (alone or in combination) in the smallest reported deletions $(2-5,8-10)$; were frequently deleted (in more than $\sim 60 \%$ of cases; refs. $1-18$ ); and have high haploinsufficiency scores (obtained from Huang et al. [ref. 19] and the Database of Chromosomal Imbalance and Phenotype in Humans using Ensembl Resources [DECIPHER]; https://decipher.sanger.ac.uk/), supporting their potential role in the syndrome.

XPO1 (also known as CRM1) is a nuclear export receptor that exports approximately 200 different cargo molecules (e.g., proteins, rRNA, small nuclear RNA [snRNA], miR, and specific mRNAs) from the nucleus to the cytoplasm. This includes molecules required for correct neuronal positioning during development (20) or synaptogenesis (21). REL (also known as c-Rel) is a transcription factor and a key component of the NF- $\mathrm{kB}$ pathway with a role in synaptic plasticity, neurogenesis, and differentiation (22, 23). USP34, a deubiquitinase, was also reported to be associated with the NF- $\mathrm{BB}$ pathway (24) in addition to having a role in the Wnt signaling pathway (25) and genomic stability (26). Finally, BCL11A, a Kruppellike transcription factor that represses transcription, is a member of the Brahma-related gene 1 (BRG1) associated factors (BAF) complex involved in the modification of chromatin structure and also has a role in regulating genomic stability (27), polarity, and migration of cortical upper layer neurons (28), as well as expression of fetal hemoglobin (4).

In order to further elucidate the genomic and clinical characteristics of $2 \mathrm{p} 15 \mathrm{p} 16.1$ syndrome, we describe 8 new subjects with deletions in the $2 \mathrm{p} 15 \mathrm{p} 16.1$ chromosome region and provide an in-depth review of all previously published cases. We also assess the function of the above-described 4 core genes ex vivo in patients' LCLs and in vivo in zebrafish. The zebrafish has recently been used as an effective model organism 
to expeditiously explore the role of a number of candidate genes within CNVs (29-31). Our study provides evidence that the knockdown of XPO1, BCL11A, and REL orthologs is sufficient to cause head and body size and shape defects in zebrafish embryos. In addition, we show that XPO1 haploinsufficiency causes impaired nuclear export in patients' LCLs. Collectively, our data strongly suggests that XPO1, BCL11A, and $R E L$ represent prime candidates for the abnormalities seen in $2 \mathrm{p} 15 \mathrm{p} 16.1$ microdeletion syndrome.

\section{Results}

Clinical and genomic findings. Summary of clinical findings of 2p15p16.1 microdeletion syndrome (25 previously reported and 8 new cases) are presented in Table 1 . Detailed clinical features of each subject are listed in Supplemental Table 1; supplemental material available online with this article; doi:10.1172/jci. insight.85461DS1. Common features include developmental delay (DD) and ID, language delay and feeding problems, hypotonia, microcephaly (or small head, 5-10 ${ }^{\text {th }}$ centile), craniofacial features (abnormal nasal root, epicanthal folds, telecanthus, ptosis, downslanting palpebral fissure, smooth and long philtrum, high, narrow palate), and distal limb anomalies (predominantly camptodactyly or metatarsus adductus) (Table 1). Structural brain abnormalities were present in $30 \%$ of cases (Supplemental Table 1). Images of some of our new cases are shown in Figure 1.

2 p15p16.1 microdeletions found in the 33 cases ranged from 0.10 to $9.57 \mathrm{Mb}$ in size and had variable breakpoints (Figure 2 and Supplemental Table 2), which contained different repeat elements at one or both sides of the CNVs (Supplemental Table 3). We excluded the presence of pathogenic mutations in genes from the intact 2 p15p16.1 region by exome sequencing of 2 cases (subject 1 in ref. 1 and new case 2). We also eliminated the effect of imprinting, as the deletions occurred on maternal and paternal chromosomes (Supplemental Table 2). In $24 \%$ of subjects, there were additional balanced chromosomal rearrangements or CNVs of unknown significance (Supplemental Table 2).

The gene content of the 2p15p16.1 deletions is shown in Supplemental Table 4, and the function of genes deleted in more than $50 \%$ of cases is shown in Supplemental Table 5. The most frequently deleted or disrupted coding genes were XPO1 and USP34 (>70\% of cases) (Supplemental Table 4). CNVs with the smallest number of deleted genes (1-4) were detected in 10 cases (Figure 2 and Supplemental Table 4). $B C L 11 A$ was the only coding gene deleted in 2 cases $(3,8)$. BCL11A was deleted along with PAPOLG in 1 patient (subject 2 in Basak et al., ref. 4) and with $P A P O L G$ and $R E L$ in the subject described by Hancarova et al. (9). Four cases had deletions of USP34 and XPO1 (Fannemel et al., ref. 10; subject 1 in Shimojima et al., ref. 2; Ronzoni et al., ref. 5; and our case 6) while two cases had larger CNVs ( 2-3 Mb) that included only FANCL and VRK2 (Prontera et al., ref. 11, and our case no. 2). In our case 2, in addition to the FANCL and VRK2 CNV, 2 smaller CNVs were detected by high-resolution array. The first CNV was in the second intron of $B C L 11 A(17 \mathrm{~kb}, 60700679-60717669$, human genome 19, hg19, assembly) and in the proximity of regulatory SNPs associated with fetal hemoglobin expression and schizophrenia (Supplemental Figure 1). The second CNV mapped to the intergenic region proximal to BCL11A (22 kb, 60828557-60851109; hg19). The extent of this CNV was refined to $27 \mathrm{~kb}$ (60828557-60855688; hg19) by quantitative multiplex PCR of short fluorescent fragments (QMPSF) using 3 primer sets. It encompassed at least half of enhancer no. 1142, which is expressed in the hindbrain in mice (Supplemental Figure 1). Additional enhancers were present in the largest 2p15p16.1 deletion segment (from the most distal to the most proximal breakpoint 55580038-66376496; hg19) (Figure 2 and Supplemental Table 6). Overall, enhancers reported on the VISTA enhancer browser (http://enhancer.lbl.gov/) suggest that chromosome 2 contains the highest number of enhancers in the genome (104 of 897 VISTA-positive and 68 of 617 brain/neuronal-expressed enhancers, of which approximately one-third are present in the $2 \mathrm{p} 15 \mathrm{p} 16.1$ region) (Supplemental Figure 2).

Given the variable breakpoints and the fact that several cases with deletions did not overlap each other (e.g., Piccione, et al. [subject 1], ref. 16; Peter, et al., ref. 8; and Hancarova, et al., ref. 9, vs. Chabchoub, et al., ref. 18; and Fannemel, et al., ref. 10), it was challenging to identify "a smallest region of overlap." Instead, we considered that genes were most likely involved in the phenotype if: (a) they were frequently deleted ( $~ 60 \%$ of cases); (b) had a haploinsufficiency score of approximately $10 \%$ or less (obtained from Huang et al., ref. 19, and DECIPHER), indicating dosage sensitivity; and (c) occurred alone or in combination in the 10 deletions with the smallest number of genes. Four genes fulfilled the criteria: XPO1, REL, $B C L 11 A$, and USP34. We analyzed these using combinations of protein expression and functional analysis in patients' LCLs, IHC staining in human brain, and in vivo zebrafish knockdown. 


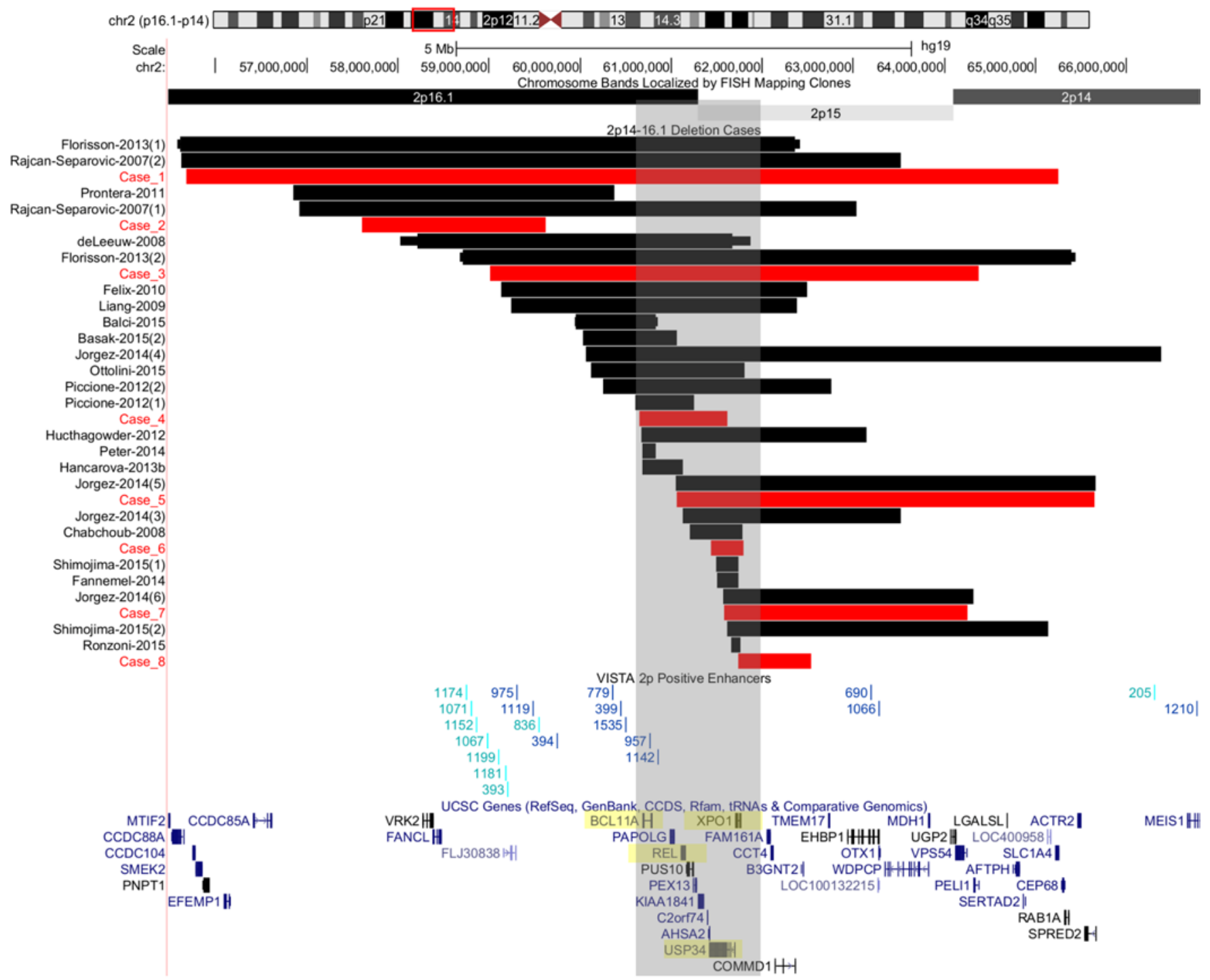

Figure 2. Summary of CNVs involving the 2p15p16.1 region. The breakpoints of the microdeletions are arranged on the basis of their start site. The black bars are published 2p15p16.1 microdeletion cases, and the red bars represent our 8 additional cases, presented here for the first time. The highlighted gray region is indicative of the 2p15p16.1 deletion region enriched in genes that are included in the smallest deletions and with the highest haploinsufficiency scores (approximately $<10 \%$ ). The 4 genes analyzed were XPO1, REL, USP34, and BCL11A and are highlighted in yellow. Enhancer elements that are located within and/or in the periphery of the CNVs are indicated with unique ID numbers and were extracted from the VISTA Enhancer Browser (http://enhancer. Iblgov/). The numbers in parentheses following the reference citation in the left-hand column indicate the subject's number.

Expression and function of 4 genes in patients' LCLs. Reduced XPO1, REL, BCL11A, and USP34 protein expression segregated with the deletion (Figure 3 and Supplemental Figure 3A). XPO1 function was further assessed by examining the nucleocytoplasmic distribution of one of its cargo proteins, 40S ribosomal protein S5 (rpS5) (Figure 4). Distribution of rpS5 was determined in cases with and without XPO1 haploinsufficiency using indirect immunofluorescence in untreated LCLs and following treatment with leptomycin B, an XPO1 inhibitor that blocks active nuclear export of XPO1 cargo molecules (Figure 4, A and B). Nuclear accumulation of rpS5 was evident in untreated LCLs from subjects with the XPO1 deletion (cases 3 and 8), compared with LCLs from a control and the subject in case 4 with intact XPO1 (Figure 4B). In fact, accumulation of rpS5 in untreated case 3 and in LCLs from 8 patients was comparable to that observed in LCLs from the control and case 4 following treatment with leptomycin B. Importantly, the level of rpS5 was unaffected in whole-cell extracts (WCEs) from XPO1-deleted and -nondeleted cases and controls, in the presence or absence of leptin B, confirming that only the cellular localization of this protein is affected in the absence of XPO1 (Figure 4C). 
A

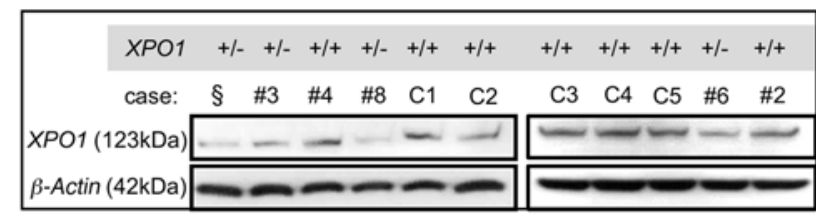

$\S$ Subject 1 in ref. 1

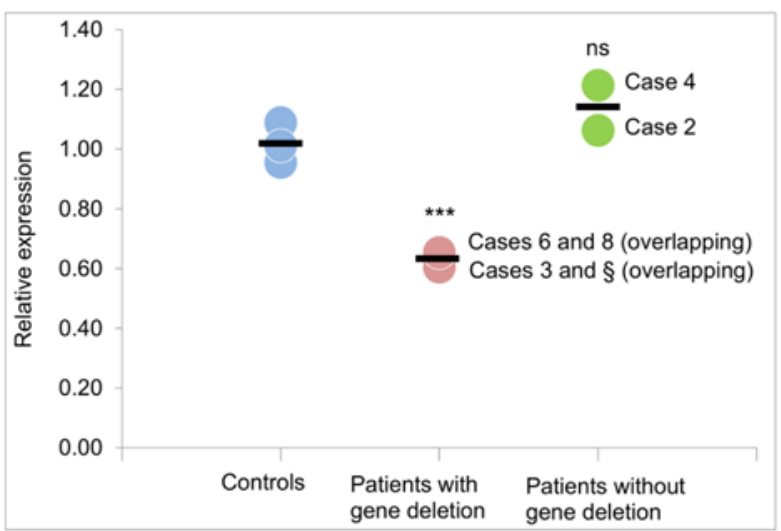

C

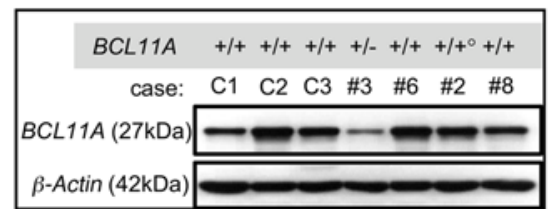

${ }^{\circ}$ Case \#2 had a small intronic BCL11A deletion

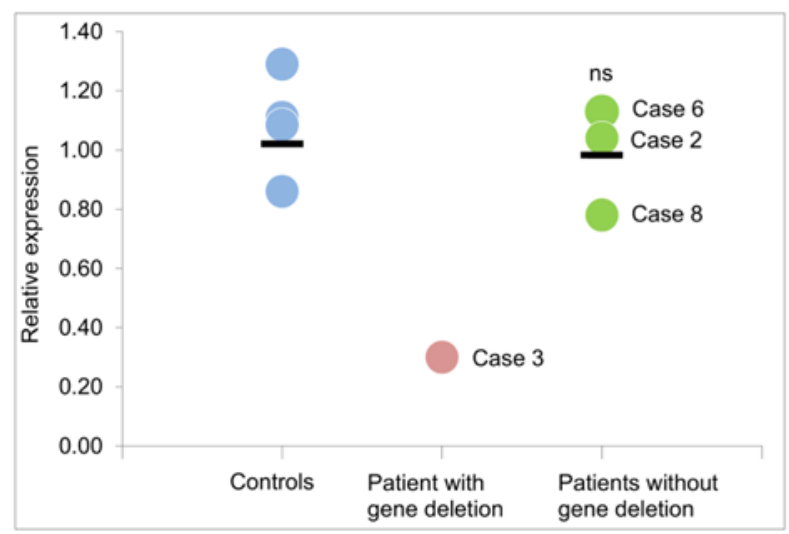

B

REL
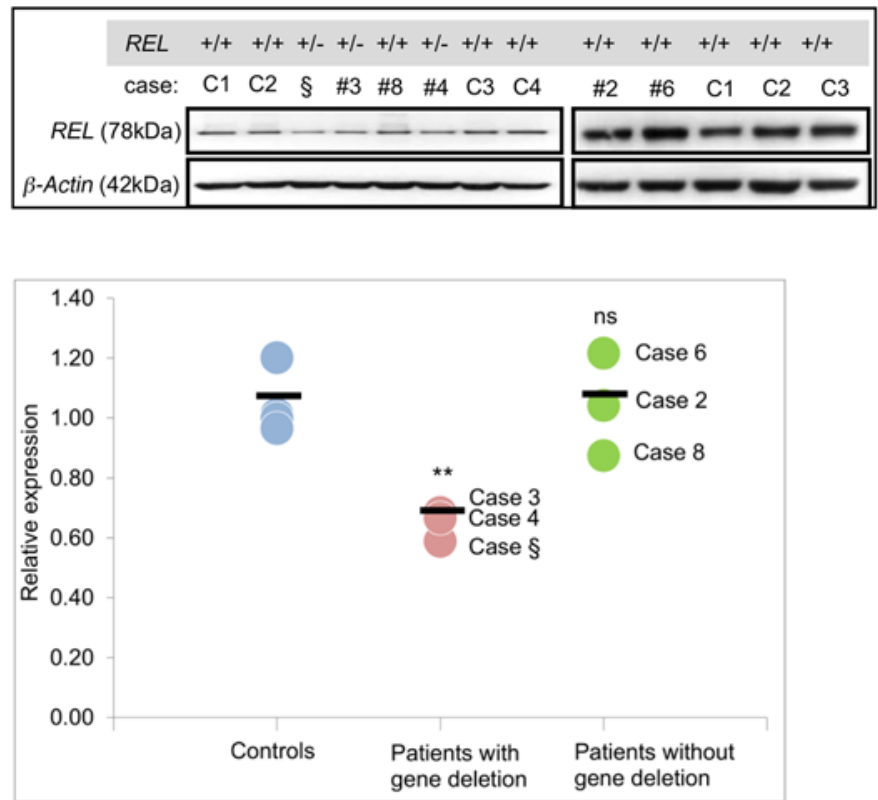

Figure 3. Protein expression analysis of XP01, REL, and BCL11A in patients' cells. Expression of (A) XP01, (B) REL, and (C) BCL11A was analyzed in the lymphoblast cell lines (LCLs) from patients (with and without gene deletions) and controls by Western blotting, and the subsequent quantitative measurement of protein density was done using GeneSnap image acquisition software (Syngene). Each data point represents the average of the ratios of normalized protein density compared with the internal loading control from independent biological replicates ( $n=3$ per individual). ${ }^{* *} P<0.001$; ${ }^{*} P<0.01$; and NS $=P>0.05$, by 2 -tailed Student's $t$ test. $\S$, Subject 1 in ref. 1. \#, case (patient) number. C1, control 1, etc.

Ubiquitin-specific protease 34, USP34, has been implicated in Wnt signaling pathway at the level of AXIN1 by opposing its $\beta$-catenin-dependent ubiquitination. Reduced USP34 expression following siRNA has been shown to lead to enhanced degradation of AXIN1 (25). While patients' LCLs haploinsufficient for USP34 showed reduced USP34 expression (Supplemental Figure 3A), AXIN1 levels were unaffected (Supplemental Figure 3B). Recently, USP34 has also been implicated in promoting DNA double-strand break repair (DSB-R) (26). The efficiency and kinetics of DSB-R can be assessed by monitoring the formation and dissipation of ionizing radiation-induced (IR-induced) p53-binding protein 1 (53BP1) foci. However, the kinetics of DBS-R as measured by IR-induced 53BP1 foci were grossly identical in cases with USP34 deletion compared with those without the deletion and compared with controls (Supplemental Figure 3C).

USP34 participates in the NF-kB pathway along with REL $(24,32,33)$. This pathway was the top pathway enriched for genes from the 2 p15p16.1 region (Supplemental Table 7) when 13 protein-coding 
A

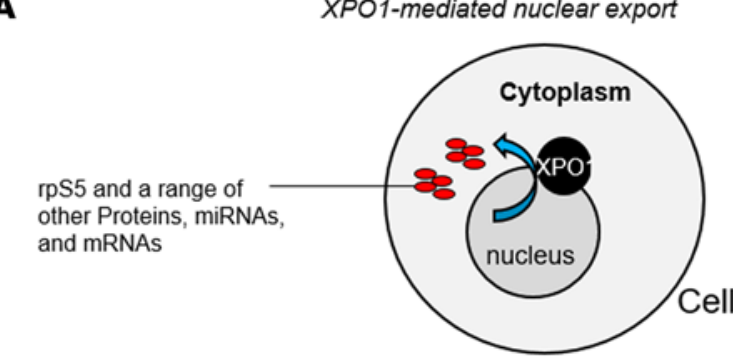

Blockage of XPO1-mediated nuclear export

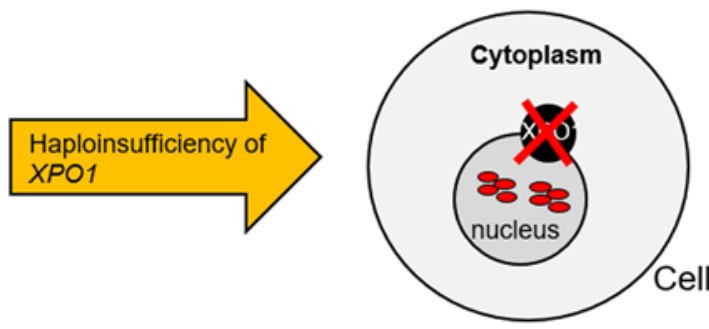

\section{B}

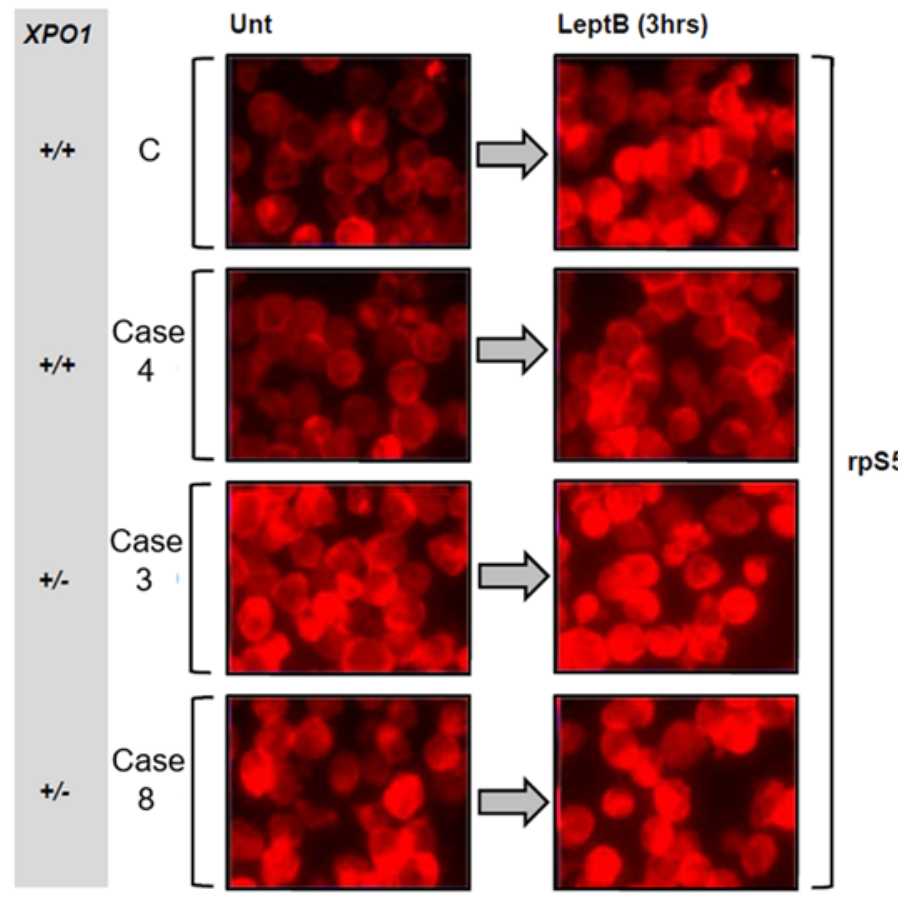

C

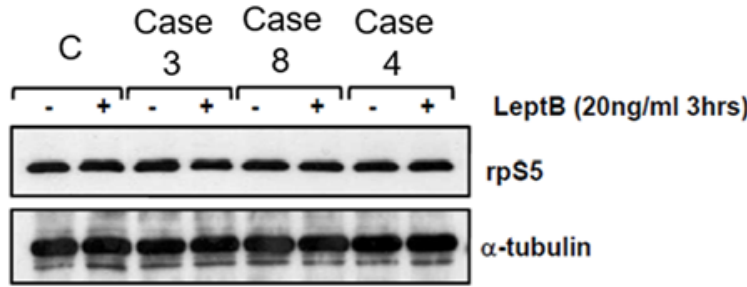

Figure 4. XPO1 dysfunction in patients' cells as determined by abnormal distribution of rpS5. (A) Normal physiological XP01-mediated export of 40S rpS5 and approximately 200 different proteins, mRNAs, and miRs from the nucleus to the cytoplasm. Suppression of XPO1 leads to blocked XP01mediated cargo and retention of proteins, mRNAs, and miRs in the nucleus. (B) Immunofluorescence images (original magnification, $\times 40$ ) of patients' LCLs showing that haploinsufficiency of XPO1 was associated with impaired nuclear export of a known cargo protein, rpS5. LCLs were either untreated (Unt) or treated with leptomycin B (LeptB), which inhibits XP01-mediated nuclear export of cargo. Untreated control LCLs (C) showed a preponderance of cytoplasmic rpS5 staining, consistent with its role at the ribosome. Upon leptomycin B treatment, nuclear accumulation of rpS5 was evident, consistent with XPO1 inhibition. A similar response was evident in LCLs from the patient in case 4 , who possessed 2 copies (+/+) of XPO1. In stark contrast, LCLs from individuals with a deletion of XPO1 (+/-) (cases 3 and 8) each exhibited significant nuclear accumulation of rpS5 when untreated, and this distribution was unaffected by treatment with leptomycin B. (C) Western blotting of WCEs from LCLs untreated (-) or treated (+) with leptomycin B showed equal rpS5 expression. LCLs, lymphoblast cell lines; WCEs, whole-cell extracts.

genes and 2 noncoding genes deleted in more than $50 \%$ of cases and recognized by WebGestalt were assessed (BCL11A, PAPOLG, REL, PUS10, PEX13, KIAA1841, LOC39803, C2orf74, AHSA2, USP34, SNOR$A 70 B, X P O 1, F A M 16 A, C C T 4$, and COMMD1) (Supplemental Tables 4 and 7). However, assessment of the $\mathrm{NF}-\kappa \mathrm{B}$ pathway in patients' LCLs showed no evidence of impaired NF- $\kappa \mathrm{B}$ signaling following induction with PMA, a specific activator of the NF- $\mathrm{B}$ pathway. We tested phosphorylation (activation) of RELA, IkB levels (which is also a cargo for XPO1; ref. 34), and nuclear accumulation of RELA, but found them to be comparable in controls and in patients' cells that had deletion of REL, XPO1, USP34, or other NF- $\mathrm{BB}-$ related genes (e.g., COMMD1) (Supplemental Figure 4 and refs. 24, 32, 33).

The effect of $B C L 11 A$ deletion on increased fetal hemoglobin expression was previously documented in patients' erythroblasts (35). Unfortunately, we were not able to obtain repeated blood samples for fetal hemoglobin expression analysis. However, for one of our patients with BCL11A deletion (case 1), hematologic findings showed increased fetal hemoglobin. 
A

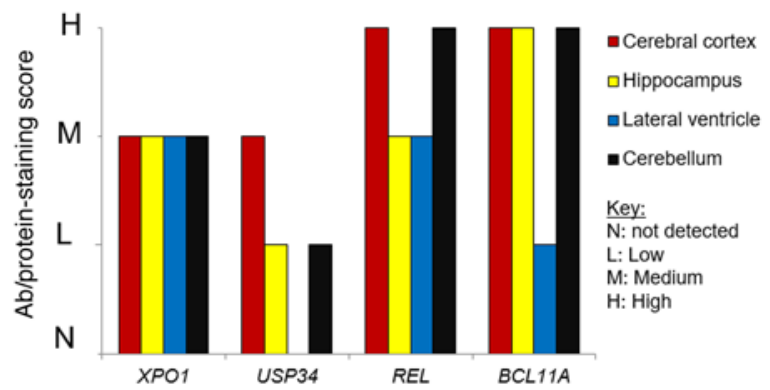

B

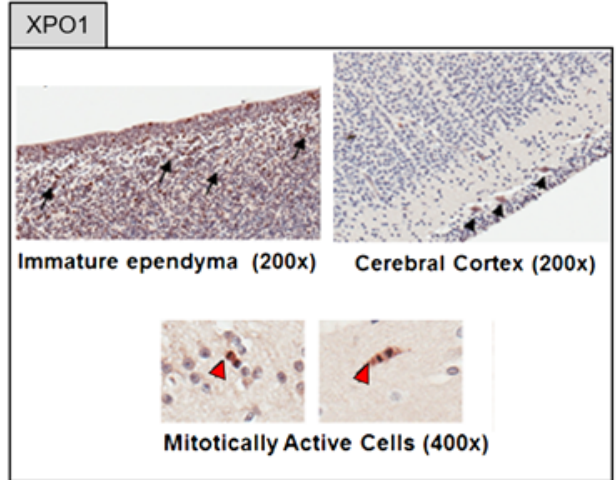

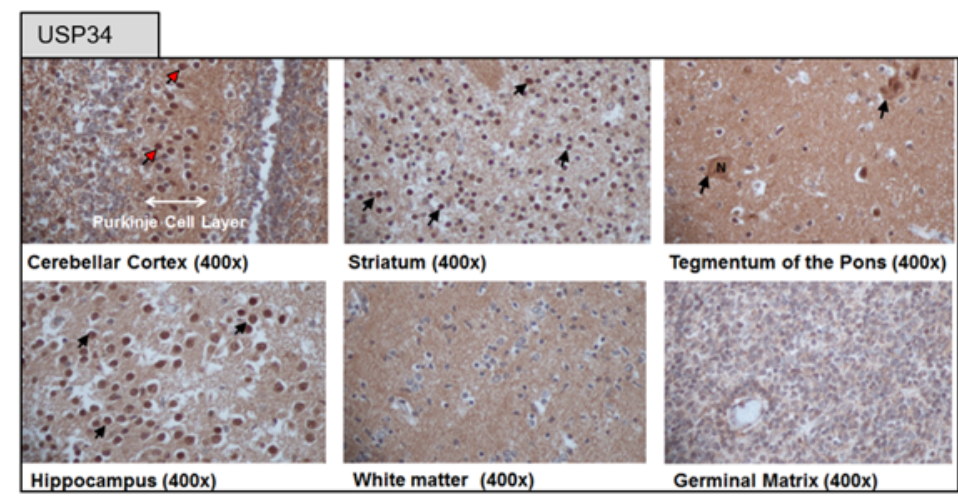

White matter $(400 \mathrm{x})$

Figure 5. Protein expression analysis of XP01, USP34, REL, and BCL11A in human brain. (A) Bar graph illustrating expression levels of all 4 genes in 4 different brain regions. Data were obtained from The Human Protein Atlas database (http://proteinatlas.org). N, not detected; L, low; M, medium; H, high. (B) Human fetal brain immunohistochemical staining performed against XPO1 and USP34. For XP01, mild positivity was seen in immature ependyma or neuroepithelium (black arrows); in the cerebral cortex, positivity was seen in Cajal-Retzius cells (black arrowheads); in immature ependymal cells undergoing mitosis (overlying the germinal matrix), positivity was stronger and associated with the mitotic spindle (red arrowheads). For USP34, strong positive staining was visible in the Purkinje cell layer of fetal brain cerebellar cortex (red arrows), while moderate positivity was seen throughout gray matter in the striatum, tegmentum of the pons, and hippocampus (black arrows); USP34 was diffusely expressed in neurons and could be seen in both the nucleus (N) and cytoplasm (black arrows) for large neurons in the tegmentum of the pons. No staining was visible in white matter or germinal layers. Original magnification, $\times 200$ and $\times 400$, as shown below each image.

Gene expression in the human brain. The Human Protein Atlas database (http://www.proteinatlas.org/) showed expression in brain and additional tissues for all 4 genes. XPO1 was most ubiquitously expressed at high or medium levels (62 of 80 tissues), followed by REL (43 of 78 tissues), USP34 (32 of 80 tissues), and $B C L 11 A$ (16 of 80 tissues). Figure 5A shows the expression level of these proteins in different brain regions. The expression levels of the 2 most frequently deleted genes, XPO1 and USP34, in control fetal and child human brains were further examined using IHC. For XPO1, IHC demonstrated mild positivity in human fetal brain (e.g., immature ependyma, Purkinje cells, inferior olive, substantia nigra, and Cajal-Retzius cells in the cortex; Figure 5B). Positivity was more intense along the spindle of some mitotically active immature ependymal cells (a pseudostratified epithelium) overlying the germinal matrix (Figure 5B), which constitute a dense stem cell population that sequentially gives rise to neuronal and glial precursors that eventually migrate out into the cerebrum. In the child's brain, mild positivity was seen only in Purkinje cells and inferior olive cells (data not shown). The staining was predominantly nuclear.

For USP34, strong positivity was seen in the Purkinje cell layer of the fetal brain cerebellar cortex, while a moderate positivity was seen in gray matter, striatum, tegmentum of the pons (both cytoplasmic and nuclear), hippocampus, caudate, putamen, and thalamus and no staining in white matter or germinal matrix (Figure 5B). In the child's brain, positivity for USP34 was more widespread and visible in both the white and gray matter, with the Purkinje layer (Bergmann glia or radial astrocytes) in the cerebellar cortex still having a strong positivity (data not shown).

Analysis of 4 genes in zebrafish. To determine the effect of deletions of the 4 human candidate genes (XPO1, USP34, REL, and BCL11A) and of 2 additional genes (FANCL and VRK2) from a CNV in a patient (case 2) with 2 small CNVs in the intron and in proximity to the $B C L 11 A$ gene, we suppressed expression of their orthologs in zebrafish embryos by knockdown with gene-specific morpholino oligomers (MO). Reciprocal Basic Local Alignment Search Tool (BLAST) analysis of the zebrafish genome showed that the 6 human 
A

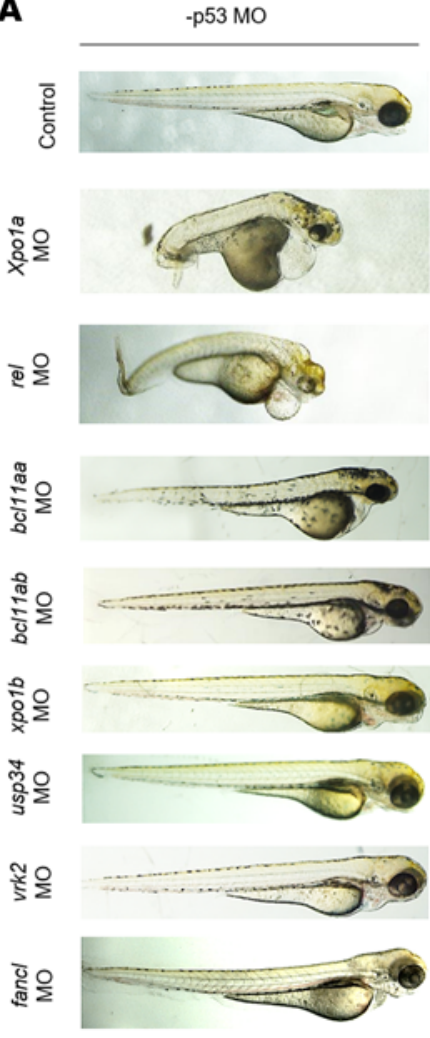

$+p 53 \mathrm{MO}$
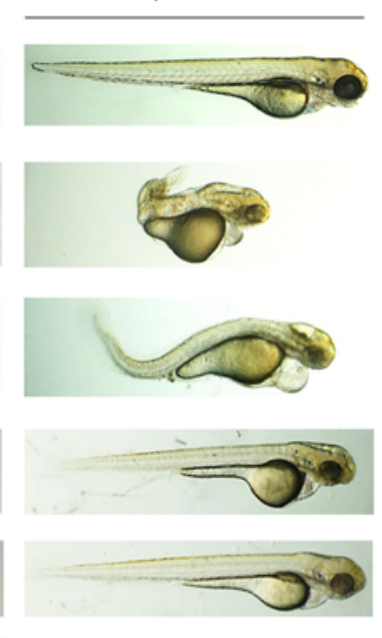

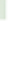

B
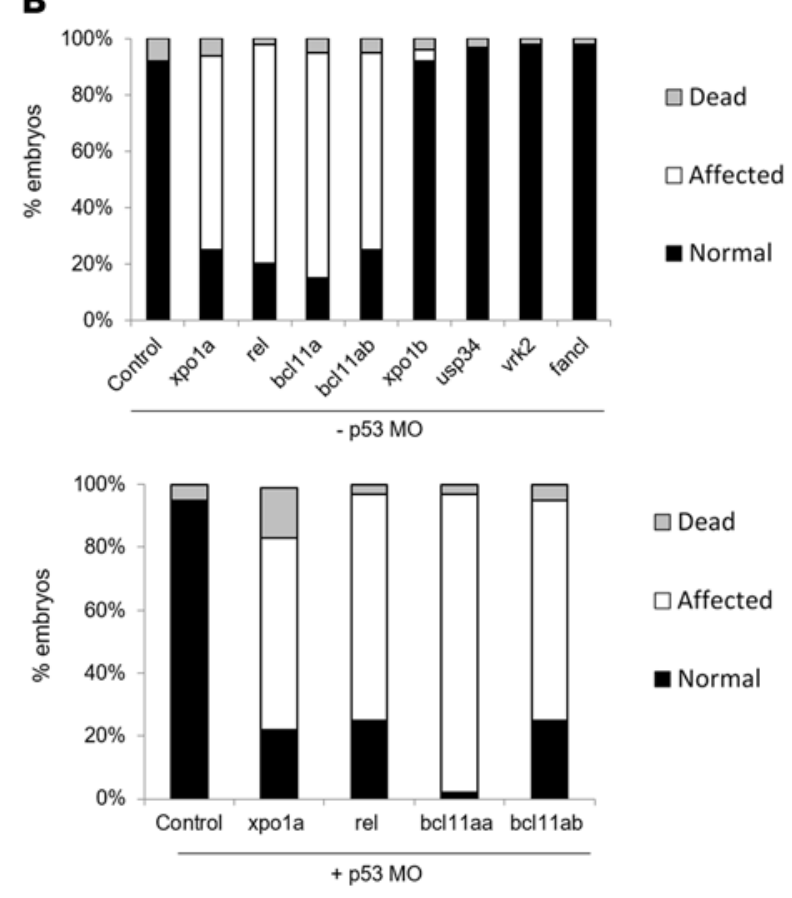

Figure 6. Knockdown of xpo1a, rel, bcl11aa, and bcl11ab genes in zebrafish causes an abnormal phenotype with head morphology and size defects. (A) Microscopic images of 3-dpf zebrafish embryos injected with gene-specific MO with or without $p 53 \mathrm{MO}$. Original magnification, $\times 50$. (B) The percentage of normal, affected, and dead fish was scored for all gene $\mathrm{MO}$ injections (+p53 $\mathrm{MO}$ and $-p 53 \mathrm{MO}$ ) at $3 \mathrm{dpf}$ and plotted. dpf, days post fertilization; MO, morpholino(s).

candidate genes had 8 orthologous genes in zebrafish. USP34, REL, FANCL, and VRK2 have 1 copy in zebrafish, while XPO1 and BCL11A each have 2 copies (xpo1a, xpo1b, bcl11aa, and bcl11ab). xpo1a and bcl11aa were considered more orthologous to the human copies, as they were similarly syntenic to usp34 on chromosome 1 and rel on chromosome 13, respectively (Supplemental Figure 5A). Protein homology of each zebrafish ortholog was also assessed and found to be highly conserved for XPO1 ( 90\%), USP34 ( 80\%), and BCL11A ( $\sim 80 \%)$, while less conserved for REL ( 50\%) (Supplemental Figure 5B).

With injection of increasing concentrations of $\mathrm{MO}(5 \mathrm{ng}, 7.5 \mathrm{ng}$, and $10 \mathrm{ng})$ for each gene, we determined that a concentration of approximately 4 to $5 \mathrm{ng}$ caused minimal embryonic toxicity (i.e., lowest lethality) yet yielded more than $60 \%$ affected embryos (Supplemental Figure 6). We found that knockdown of 4 of the 8 genes, xpola, rel, bcl11aa, and bcl11ab, consistently resulted in visible phenotypic abnormalities of the head and body in more than $70 \%$ of injected embryos at approximately $5 \mathrm{ng} \mathrm{MO}$ (or lower), with and without knockdown of $p 53$, used to minimize MO-induced apoptosis and off-target effects (Figure 6 and Supplemental Figure 6). No visible phenotypic abnormalities were observed for the other 4 genes, usp34, fancl, vrk2, and xpo1b, at a high MO concentration of $10 \mathrm{ng}$ at 1 and 3 days post fertilization (dpf) (Figure 6 and Supplemental Figure 6).

Significant microcephaly ( $P<0.0001, n=50$ per injection) was consistently noted for 3 genes, xpo1a, $b c l 11 a a$, and rel, when suppressed alone or in combination with $p 53$ (Figure 7). As a secondary parameter of their head abnormalities, we measured the size of their otic vesicles (developing inner ear). Significant otic vesicle reduction $(P<0.0001)$ was observed in rel, bcll1aa, and bcl11ab morphants, whereas the otic vesicle size in xpola morphants was comparable $(P=0.1619)$ to that seen in controls (Figure 7$)$. Knockdown of either xpo1a or rel caused a dysmorphic, curved body in zebrafish, while bcl11aa and bcl11ab morphants did not have the severely affected body shape. However, measuring the length of 5 tail somites in bcl11aa morphants suggested a significant reduction in size compared with that observed in controls (Figure 7). Eyes and fins were also noted to be abnormal and visibly much smaller for almost all injected fish compared with control eyes and fins. Head and body size measurements for the other noncausative genes, usp34, fancl, vrk2, and xpo1b, were comparable to those of controls (Supplemental Figure 7). 

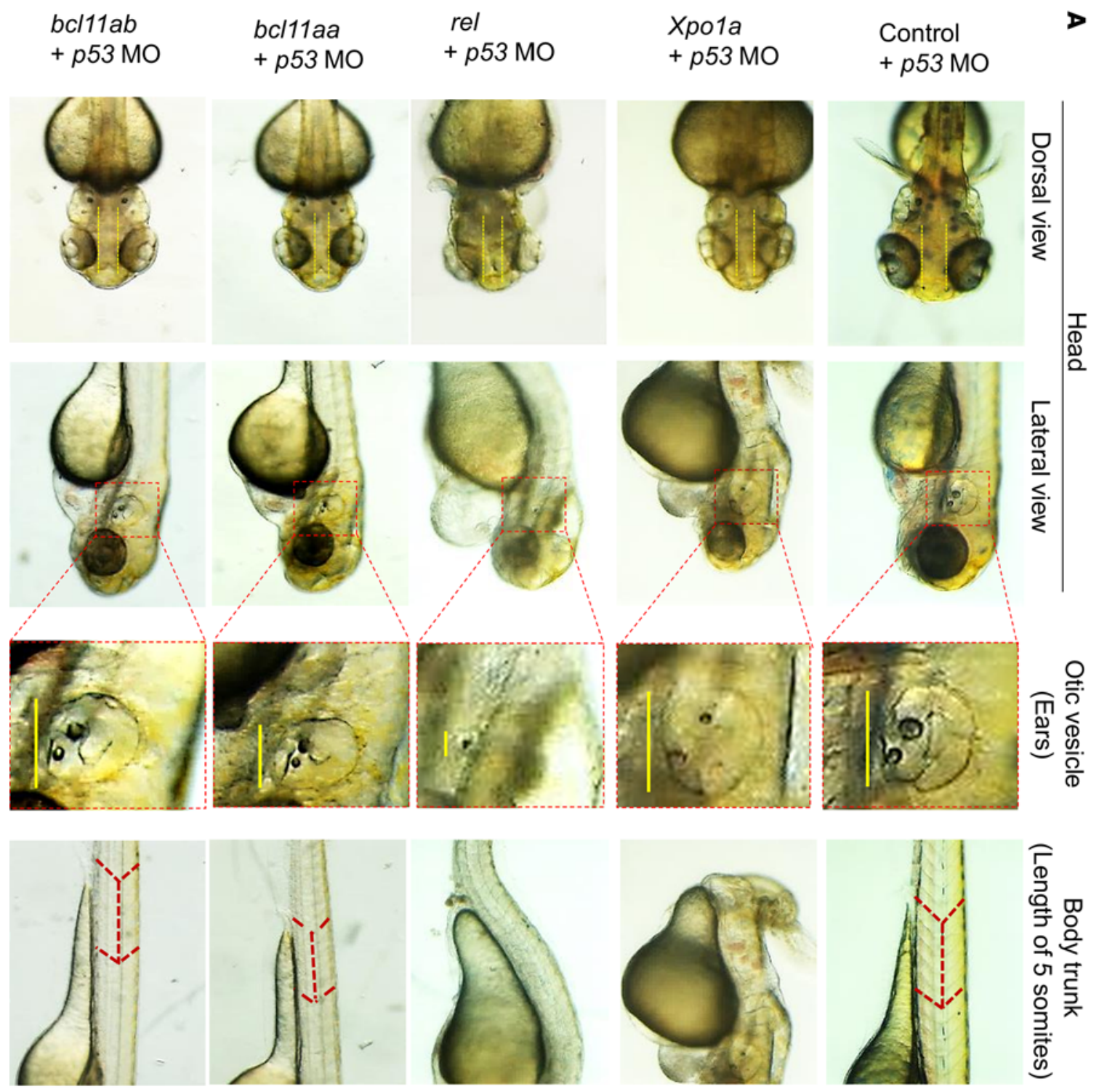

$\boldsymbol{\omega}$
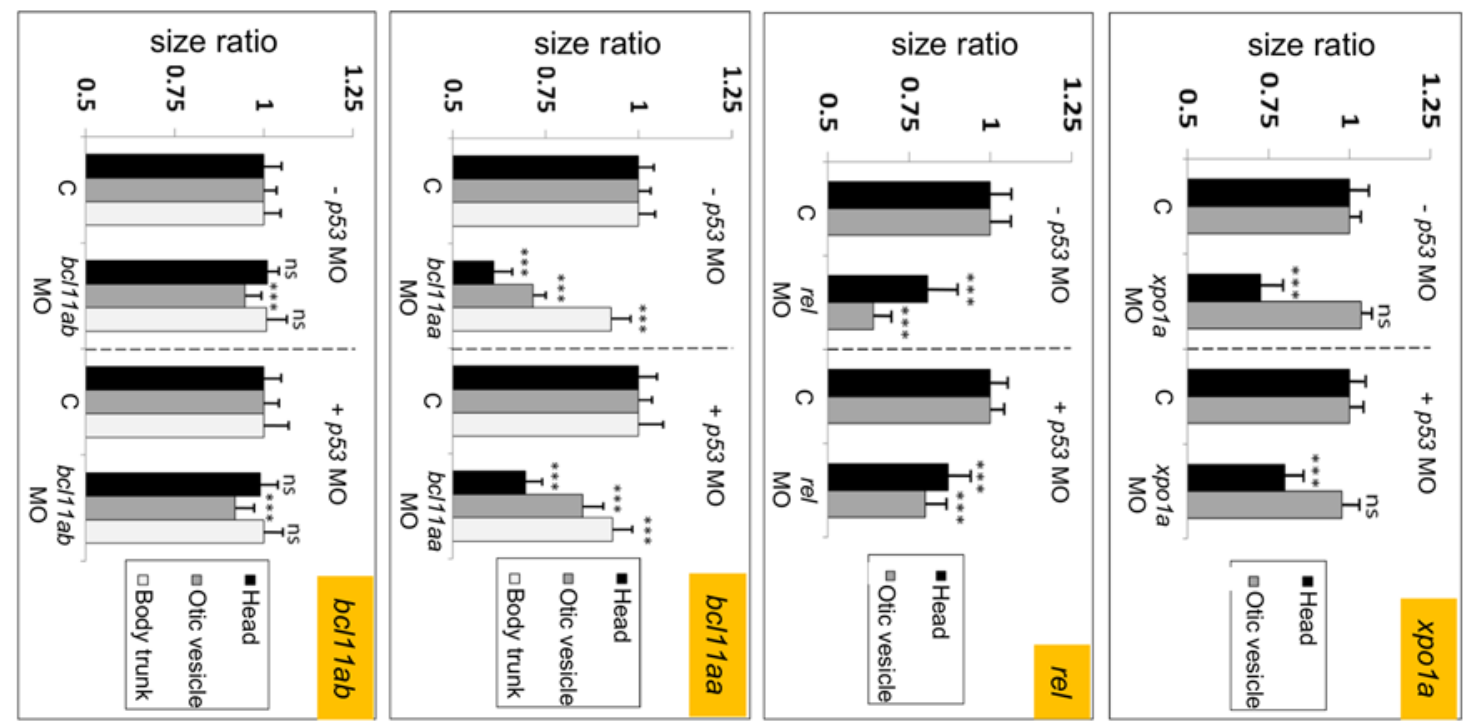
Figure 7. Measurement of head, otic vesicle, and body size in affected zebrafish embryos. (A) Microscopic images and (B) size measurements of the head (distance between the eyes), otic vesicle, and body trunk (distance between 5 tail somites) of 3-dpf embryos injected with the 4 causative gene MO: xpo1a, rel, bcl11aa, and bcl11ab. Microcephaly was observed for 3 gene morphants compared with those of controls (xpo1a, rel, and bcl11ab). Otic vesicle size was severely reduced for rel, moderately reduced for bc/11aa and bcl11ab, and comparable to that of controls for xpo1a knockdowns. The body trunk was fully dysmorphic and curled for xpo1a and rel morphants, while measurement for bc/11aa and bc/11ab morphant embryos was possible. Smaller eyes and fins for all genes were noted. Original magnification, $x 115$. (B) Measurements for both $-p 53$ and $+p 53$ M0 injections were normalized to the sizes obtained in controls, and the ratios were plotted. Data represent the mean \pm SD of 3 independent injections. $n=50$ embryos per gene per injection. ${ }^{* *} P<0.0001$ and NS $=P>0.01$, by 2 -tailed Student's $t$ test for significance of differences in measured sizes between each gene morphant and controls. MO, morpholino(s).

In addition to head and body structure anomalies assessed at $3 \mathrm{dpf}$, analysis of 1-dpf embryos suggested structural brain abnormalities for morphants targeting xpo1a, rel, bcl11aa, and bcl11ab (Figure 8). Although the forebrain and midbrain of the 1-dpf xpola and rel morphants seemed comparable to those of the controls, the hindbrain ventricle for both genes was abnormally developed and not properly expanded in approximately $50 \%$ to $70 \%$ of knockdowns compared with controls, as the ventricle walls were not stretched enough to allow brain cavities to form (Figure 8). This feature has previously been reported for suppression of other genes that have a role in brain and neural development (36), supporting the role of xpola and rel in neurodevelopment. The most dramatic brain abnormality was noted in $b c l 11 a a$ and bcl11ab morphants, in which the brain lacked differentiation of structures or expansion and resembled an underdeveloped neural tube. Shrinkage of whole-brain volume was detected for these 2 genes in almost all of the injected fish embryos (>90\%) (Figure 8 ). The overall development of the fish was therefore significantly impaired for xpola, rel, bcl11aa, and bcl11ab, but the abnormalities also demonstrated gene specificity. Features such as heart edema as well as swim bladder anomalies were considered as nonspecific effects.

The splice-blocking MO (SB-MO) knockdown of all genes (except rel) by blockage of their normal pre-mRNA splicing was confirmed by reverse transcription PCR (RT-PCR) of total RNA extracted from 1- to 3-dpf MO-injected fish. Full or significant absence of normal transcript and/or the presence of truncated abnormally spliced transcripts was visible after gel electrophoresis of PCR products for each gene (Supplemental Figure 8, A and B). For rel only, the knockdown was confirmed by translation-blocking MO (TB-MO) injection and subsequent Western blotting of the protein extracted from the morphant embryos (Supplemental Figure 8C), as RT-PCR confirmed that 2 independently designed SB-MO for this gene failed to target and block the expression.

Therefore, the zebrafish developmental defects produced as a result of the knockdown of the orthologous copies of human XPO1, REL, and BCL11A support the role of these genes in the 2p15p16.1 microdeletion syndrome phenotype and identify them as 3 key dosage-sensitive genes "driving" the abnormal development.

\section{Discussion}

We describe 8 new patients with 2p15p16.1 microdeletions ranging from 0.36 to $9.57 \mathrm{Mb}$ and with overall features in keeping with 2 p 15 p16.1 microdeletion syndrome. The severity of the phenotype could not be correlated with the size of the CNV. In fact, a comparable number of phenotypic abnormalities (44 vs. 34) determined by the same geneticist to minimize variability were noted in 2 cases with the largest and smallest deletions in our cohort (9.57 Mb in case 1 and $0.36 \mathrm{Mb}$ in case 6, respectively). Furthermore, we noted that similar features were reported for patients with a different gene content. For example, our case 6 (with XPO1/ USP34 deletion) and the case reported by Hancarova et al. (9) (with BCL11A, REL, and PAPOLG deletions), both had prenatal and postnatal growth delays, microcephaly, hypotonia, spasticity of legs, downslanting palpebral fissures, dysplastic ears, abnormal hematologic findings, and digital anomalies. This suggests that several critical developmental genes in the $2 \mathrm{p} 15 \mathrm{p} 16.1$ deletion region could contribute to the phenotype.

We selected 4 genes, XPO1, BCL11A, USP34, and REL, for follow-up by modeling knockdown of their orthologs in zebrafish and functional assessment in patients' LCLs on the basis of their frequent deletions in 33 published cases and inclusion in the CNVs with the smallest number of genes. These genes were also deleted, alone or in combination, in approximately $70 \%$ of the smaller $(<1 \mathrm{Mb})$ CNVs cataloged in the DECIPHER and International Standards for Cytogenomic Arrays (ISCA) Consortium databases. The absence of recurrent deletions overlapping the 4 genes in healthy controls cataloged in the Database of Genomic Variants (DGV)(http://dgv.tcag.ca/) supports the pathogenicity of these genes. 
A

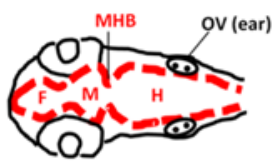

B

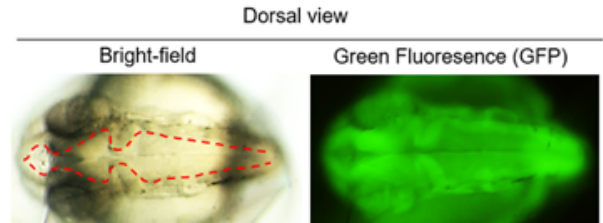

xpo1a

+ p53 MO

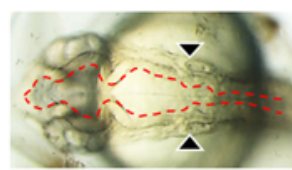

rel

+ p53 MO

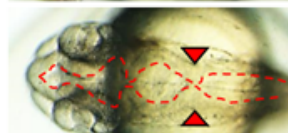

bcl11aa
+ p53 MO

c11ab

+ p53 MO
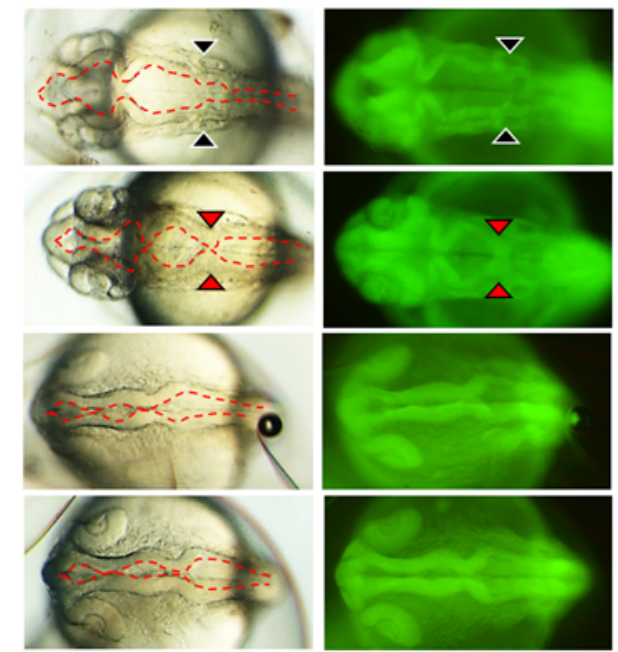

C

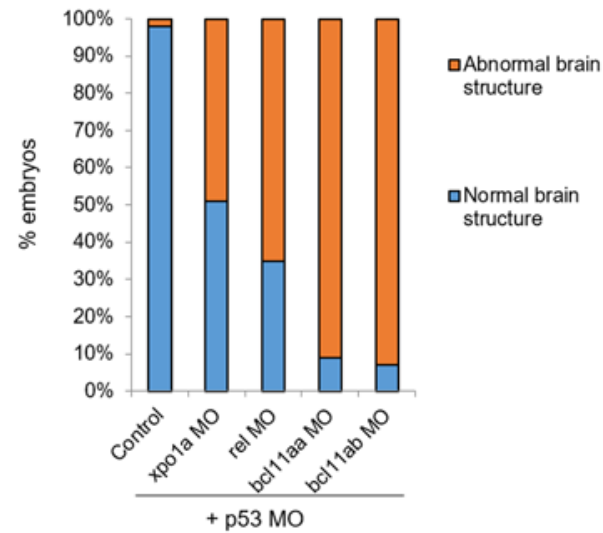

Figure 8. Brain structural anomalies in affected zebrafish embryos. (A) Representative depiction of the forebrain (F), midbrain (M), hindbrain (H), midbrain-hindbrain boundary (MHB), and otic vesicle (OV) structures in 1-dpf embryos. (B) Bright-field and GFP microscopic images of the brain structure of 1-dpf embryos injected with the 4 causative genes, xpo1a, rel, bc/11aa, and bc/11ab (all +p53 MO). Moderate-to-severe abnormal brain cavity formation was noted in all injected fish. xpo1a knockdown caused a mild narrowing of the hindbrain (indicated with black arrowheads), rel suppression led to hypoplastic hindbrain cavity development, which was manifested with pinched-off hindbrain (indicated with red arrowheads), while knockdown of both bc/11aa and bc/11ab led to shrunken brain volume with poor development of all compartments. Original magnification, $\times 115$. (C) The percentage of observed brain phenotypic abnormalities for each gene was scored. $n=50$ embryos per gene. MO, morpholino(s).

$X P O 1$ appears to be the strongest candidate for the syndrome. The elevated nuclear accumulation of rpS5 in LCLs from patients with XPO1 haploinsufficiency supports the previous observation that the biogenesis and subsequent nuclear export of ribosomal constituents is dependent on XPO1 (37) and suggests that its inhibition could lead to repression of ribosomal biogenesis, downregulation of translation, and apoptosis, as previously shown with leptomycin B-induced inhibition of XPO1 (38). Knockdown of xpo1a in zebrafish resulted in abnormal development including small head and brain abnormalities, further implicating this gene in the phenotype and supporting the finding that the vast majority of patients with $X P O 1$ haploinsufficiency show microcephaly. Only 4 of the 25 subjects with XPO1 deletion (our case 8 and the patients described in refs. 5,15 , and 18 ) had a normal head size, although they had other cranial abnormalities including a high forehead (18) or structural brain abnormalities (5). The role of XPO1 in the syndrome is also supported by its widespread expression in both fetal and child brains, along with its established roles in the transport of molecules relevant for synapse formation (e.g., neurogenin 3, ref. 21), neuronal positioning during brain development (20), and stabilization of centrosomes and mitotic spindles of the dividing cells (39). In humans, XPO1 SNPs were linked to autism (40), mutations with genomic instability in cancer (41), and overexpression in multiple sclerosis (MS) (42). To date, patients with isolated deletion of XPO1 were not reported, although 4 cases had a deletion containing only XPO1 and USP34 (subject 1 in Shimojima et al., ref. 2; Fannemel et al., ref. 10; Ronzoni et al., ref. 5; and our new case 6), with all patients having microcephaly and/or structural brain abnormalities.

The role of the second most commonly deleted gene in the syndrome, USP34, in the 33 subjects remains uncertain, as no cellular phenotype for pathways previously associated with USP34 dysfunction (Wnt- and $\mathrm{NF}-\mathrm{kB}$-signaling pathways as well as genomic instability) was identified in cells with the deletion, despite 
reduced expression of USP34 in LCLs from patients with USP34 haploinsufficiency. Similarly, there was no phenotypic effect in zebrafish with usp34 knockdown. Furthermore, 1 of the 4 small CNVs containing USP34 in patients from DECIPHER was maternal in origin (DECIPHER patient no. 314299). As the function of this gene becomes better known, its role in the syndrome may be elucidated.

$B C L 11 A$ is an intriguing candidate for phenotypic abnormalities on the basis of reports of patients with both larger and smaller deletions of $B C L 11 A$ alone $(3,8)$. We showed that knockdown of both $B C L 11 A$ orthologs in zebrafish resulted in microcephaly and significant structural brain abnormalities, supporting the role of BCL11A in the 2p15p16.1 microdeletion phenotype. BCL11A inhibits fetal hemoglobin expression $(4,43)$, which was increased in patients with $B C L 11 \mathrm{~A}$ deletion, and our case no. 1 . It is of interest to note that even without an obvious deletion of $B C L 11 A$, fetal hemoglobin expression was increased in a patient with isolated $F A N C L$ and $V R K 2$ deletions $(11,35)$, possibly suggesting an effect of the distant deletion on the function of BCL11A. Regulatory SNPs were previously reported in the enhancer sequence that lies in the second intron of $B C L 11 A$ and were associated with increased expression of fetal hemoglobin as well as with schizophrenia and attention deficit hyperactivity disorder (ADHD) (4). Similar to the patient described by Prontera et al. (11), our case no. 2 patient had a deletion that included only FANCL and VRK2 as coding genes, however, higher-resolution array detected 2 smaller CNVs in the second intron of $B C L 11 A$, and in the proximal region to BCL11A partially overlapping 1 enhancer element. Unfortunately, the role of these 2 small CNVs remains uncertain, as no blood was available for assessment of fetal hemoglobin-containing erythroblasts from this patient, and BCL11A expression was normal in the patient's LCLs. FANCL and $V R K 2$ appear less likely to have a significant role in the phenotype of our case no. 2, given the normal development observed following knockdown in zebrafish and given the finding that 1 of the 5 DECIPHER patients with deletions in the VRK2/FANCL gene region (patient no. 274140) inherited the VRK2/FANCL deletion from a normal parent. We believe that the additional CNV in our patient no. 2, from the pericentromeric region of chromosome 12 (12p11.21q11), is unlikely to explain the characteristic $2 \mathrm{p} 15 \mathrm{p} 16.1$ microdeletion syndrome features observed in this patient (e.g., straight eyelashes, ptosis, downslanting palpebral fissures, and microcephaly), given the function of the genes involved in the 12p11.21q11 CNV (Supplemental Table 2).

Finally, we implicate $R E L$ in the $2 \mathrm{p} 15 \mathrm{p} 16.1$ microdeletion phenotype, given the reduced protein expression detected in LCLs from patients with this deletion and given the structural brain abnormalities, abnormal growth, and dysmorphism observed following knockdown in zebrafish. This is in keeping with the previously noted neuroprotective and antiapoptotic role of $R E L$ in the NF-kB pathway and its role in hippocampal long-term plasticity and memory formation $(23,44,45)$.

Our findings suggest that the abnormal phenotype caused by $2 \mathrm{p} 15 \mathrm{p} 16.1$ deletions could be a result of a deletion of at least 1 of the 4 genes; however, we cannot exclude the role of other coding or noncoding sequences from this region. Nine additional coding genes were deleted in more than $50 \%$ of cases. These include PUS10, PEX13, C2orf74, AHSA2, FAM161A, CCT4, COMMD1, KIAA1841, and PAPOLG, but we consider them less likely to be the drivers of the phenotype, given that they tend to occur only in larger CNVs. Of these latter 9 genes, disease-causing mutations were noted in The Human Gene Mutation Database (HGMD) (http://www.hgmd.cf.ac.uk/) for FANCL (Fanconi anemia), PEX13 (Zellweger syndrome), FAM161 (retinal abnormalities), and COMMD1 (elevated urinary copper), and all were reported to be autosomal recessive conditions. Only the autosomal recessive genes COMMD1, PEX13, and CCT4 were associated with neurodevelopmental abnormalities in mouse- or rat-KO models (46-48); however, we excluded the presence of deleterious mutations in their intact alleles by exome sequencing.

It is interesting to note the functional relationship between a number of genes from the $2 \mathrm{p} 15 \mathrm{p} 16.1$ region supported by colocalization of pairs of orthologs of human XPO1/USP34, BCL11A/REL, and FAN$C L / V R K 2$ in zebrafish. Furthermore, XPO1 exports protein products of $2 \mathrm{NF}-\kappa \mathrm{B}$ genes from 2p15p16.1 region, COMMD1 (33) and USP34 (32), and USP34 interacts with REL (c-Rel) (49). The NF-kB pathway was the top enriched pathway for 15 coding and noncoding genes deleted in more than $50 \%$ of the 33 cases; however, its functionality appeared intact in LCLs from patients carrying deletions of the above genes. It

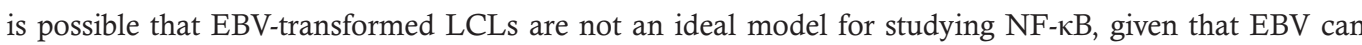
activate this pathway and possibly mimic its native state in patients' cells (50). Dysfunction of several genes from 2p15p16.1 was also associated with genomic instability (BCL11A, ref. 27; USP34, ref. 26; and XPO1 ref. 39), however, one aspect of genomic stability (i.e., the repair of IR-induced DNA breaks) in LCLs from patients with deletions was comparable to that seen in controls. 
In addition to the coding genes, a previous study (7) raised the question of the role of enhancers in this region. We noted that chromosome 2 has the highest number of positive human enhancer elements (i.e., those with tissue-specific expression and activity confirmed in vivo in mice; ref. 51) in the genome reported in the VISTA database and one-third of the enhancers from chromosome 2 are in the deleted region. However, other than the role of an intronic enhancer for $B C L 11 A$, the importance of these enhancers and their possible roles in the transcriptional regulation of the genes from the $2 \mathrm{p} 15 \mathrm{p} 16.1$ region or beyond are as yet unknown.

The recurrence of deletions in the $2 \mathrm{p} 15 \mathrm{p} 16.1$ region of variable size is intriguing, and we demonstrated the presence of different types of repeats at the breakpoints as well as an absence of low-copy repeats (LCRs) (40). Constitutional duplications in the region were also reported in 8 DECIPHER cases and 1 recently reported case (chr2:60150427-61816209, hg19) (52) that had no consistent abnormalities or evidence of a mirror image phenotype (e.g., macrocephaly). Further evidence of genomic instability in the same region exists in cancer, leading to loss, gain, or amplification of 2 p15p16.1 genes in approximately $30 \%$ of cases of lymphoma $(53,54)$. It is interesting to note that cancer-related abnormalities in the $2 \mathrm{p} 15 \mathrm{p} 16.1$ region were reported to coincide with fragile site, aphidicolin type, common, fra(2)(p13) (or FRA2E) (55).

We conclude that the $2 \mathrm{p} 15 \mathrm{p} 16.1$ region is very complex genomically, but the phenotype of patients strikingly similar, considering the variable genomic sizes of the deletions. The phenotypic consequences of knocking down the 4 genes identified here have not, to our knowledge, been previously reported in zebrafish and highlight the possibility that multiple genes contribute to 2 p15p16.1 deletion syndrome. XPO1 represents a particularly strong candidate, but the existence of patients without deletion of this gene indicate that there are other causes of the abnormal phenotype. These causes could include other potent developmental genes, their interaction and common pathway disruption, as well as the involvement of regulatory elements from this region. Very fine molecular dissection of the region to search for additional functional defects in patients' cells, sequence analysis of breakpoints, and in-depth analysis of regulatory elements could perhaps shed more light on the complexities of the region and the association of these factors with abnormal development in the future.

\section{Methods}

Clinical and genomic data acquisition. Since our report of the initial 2 patients in 2007, we have recruited 8 additional patients with 2p15p16.1 microdeletions (cases 1-8) to our research cohort. Microdeletion breakpoints for all cases here were from the human genome 19 assembly (hg19, University of California Santa Cruz [UCSC] database). UCSC genome browser customization tool was used to illustrate the CNV characteristics (including breakpoints, size, and gene content) in the 33 patients. International databases cataloging CNVs in patients (DECIPHER, ISCA, and the European Cytogeneticists Association Register of Unbalanced Chromosome Aberrations [ECARUCA]) as well as in healthy controls (DGV; http://dgv. tcag.ca/) were used to assess additional CNVs in the $2 \mathrm{p} 15 \mathrm{p} 16.1$ region.

DNA and chromosome microarray analysis. DNA was extracted from peripheral blood using the ArchivePure DNA blood kit (5 PRIME, \#2300740). Chromosome microarray analysis for new cases was performed using commercial arrays (e.g., Signature Genomics), and, whenever possible, high-resolution 2.7 $\mathrm{M}$ or Cytoscan array analysis was repeated according to Affymetrix protocols and as previously described (56). DNA from the case reported previously by Chabchoub et al. (18) was obtained courtesy of Thomy de Ravel (University of Leuven, Leuven, Belgium) for higher-resolution array analysis.

Confirmation of small CNVs using QMPSF. QMPSF was performed to confirm array findings for the 2 small deletions in intron 2 and around the $B C L 11 A$ gene detected in the new case 2 using previously described protocols (57). Briefly, short fragments were PCR amplified from genomic DNA using primers labeled with a fluorescent dye and fragment sizes analyzed by capillary gel electrophoresis. Primers were designed for intron 2 of the BCL11A region and 3 regions in and around enhancer 1142 in the proximity of $B C L 11 A$ and were as follows: intronic: forward, 5'-TTCTAGTGCTTTGGGCGAGT-3'; reverse, 5'-GGAATGCTGCAGTTGTCAGA-3' (60710726 - 60710922); within the enhancer: forward, 5'-ACATGGCCAGACCTGAAAAC-3'; reverse, 5'-CAGAAAGGCTGAACCCTGAG-3' (60855485 - 60855688); distal to the enhancer: forward, 5'-TGCATGAGGACATTGGTGAT-3'; reverse, 5'-CTCAAGGGAAGGAGCTGTTG-3' (60850362 - 60850585); distal to the enhancer: forward, 5'-GAAGGTTCCCACGTTTTGAA-3'; reverse, 5'-TTATTTGCCCCCAGTGAGAG-3' (60835872 - 60836064). 
Whole-exome sequencing analysis. Whole-exome sequencing was performed for the DNA sample obtained from 2 individuals with a 2p15p16.1 deletion. One of them had a large deletion encompassing all 4 genes (subject 1 reported in ref. 1), and the other case (new case 2) had a CNV containing FANCL and VRK2, a $\mathrm{CNV}$ in intron 2 of $B C L 11 A$, and a CNV proximal to it. For subject 1 (1), sequencing was performed by Otogenetics with Agilent's 50-Mb SureSelect exome capture kit and HiSeq2000, and the Ion Torrent protocol was used for the patient in case 2. Reads were aligned to genome assembly hg19, and files containing variant calls were generated using an in-house pipeline. The files with annotated calls were then imported into Golden Helix SNP and Variation Suite 7.7.8 for further analysis. Briefly, variant calls were filtered using quality scores of the sequence data as follows: read depth greater than or equal to 10 , genotype quality scores of 10 or higher, and an alternate allele frequency of greater than or equal to $25 \%$. Variants were then restricted to the chromosomal region overlapping the $2 \mathrm{p} 15 \mathrm{p} 16.1$ deletion, after which they were filtered to eliminate variants present in publicly available databases including 1000 Genomes (1KG, 2012-04-26); the National Heart, Lung, and Blood Institute Grand Opportunity Exome Sequencing Project (NHLBI ESP6500 v2); and the Exome Aggregation Consortium (ExAC) (http://exac.broadinstitute.org/), at frequencies of greater than or equal to $1 \%$ of the population.

Mutations reported in the HGMD (http://www.hgmd.cf.ac.uk/) were extracted for the most frequently deleted genes from the $2 \mathrm{p} 15 \mathrm{p} 16.1$ region.

CNV parent-of-origin microsatellite analysis. To determine whether the $2 \mathrm{p} 15 \mathrm{p} 16.1$ deletion occurred on the maternal or paternal chromosome, a microsatellite (24xTA, genomic size 48 ) mapping to $2 \mathrm{p} 15$ (chr2:61,755,869-61,755,916; hg19) was selected using the UCSC genome browser. The sequences for the forward and reverse primers were: forward, D2S_XPO1_24TA: 5'-AGCCAAGATTCCCCAAGAAT-3' and reverse, D2S_XPO1_24TA: 5'-AAGCCATTGCTTTTTGTCAAT-3'. PCR products were separated by capillary electrophoresis on an ABI PRISM 310 Genetic Analyzer, and peak size and area were determined with GeneScan Analysis Software, version 3.1.2 (ABI).

DNA repeated elements by RepeatMasker. Repeated elements (RMSK elements) flanking each deletion were identified using RepeatMasker (http://www.repeatmasker.org/). In order to do this, a user-generated bed file containing 23 2p15p16.1 deletion regions, using human genome assembly GRCh37/hg19, was uploaded into Galaxy v 1.0.0. Regions flanking the deletions (500 bp upstream and downstream) were generated using the Galaxy Tool (https://usegalaxy.org/) by selecting "operate on genomic intervals" and "get flanks" from the pull-down menu. The following input parameters were entered: Select data 5: 2p15p16.1 23 cases, Region: Whole feature, Location of the flanking region(s): Both, Offset: 0, Length of the flanking region(s): 500. The 46 flanking regions were then joined with the Repeating Elements by RepeatMasker dataset taken from the UCSC (UCSC Main on Human: rmsk [genome], which contains approximately $5,400,000$ regions in interval format, hg19) using the join function (INNER JOIN) in the Galaxy Tool with minimum overlap of $1 \mathrm{bp}$.

Enhancer elements data mining. Enhancer elements in the 2p15p16.1 deletion region were extracted from the VISTA Enhancer Browser (http://enhancer.lbl.gov/) using the genomic position from the most distal to the most proximal breakpoints in all 33 cases (chr2:55580038-66376496; hg19) for human-only enhancers. The VISTA Enhancer Browser was also used to find and compare the distribution of all positive enhancer elements in the human genome (total of 897) among different chromosomes. For extraction of brain- and neural-expressed enhancers, only those with expression patterns in the neural tube, cranial nerve, forebrain, midbrain, hindbrain, trigeminal V (ganglion, cranial), dorsal root ganglion, and neural crest-derived and facial mesenchyme were selected (total of 617), and the genomic distribution of these enhancers was assessed. The distribution of brain- and neural-expressed enhancers in each chromosomal band over the total number of enhancers on chromosome 2 was also determined (only bands with at least 4 enhancers were considered in this analysis).

Functional analysis of 4 genes in patients' cells. Patients' LCLs were established as previously described (58). Western blotting was performed according to standard protocols (58), using the following Abs: antiCRM1/XPO1 (Novus Biologicals, NB100-56493); anti-Ctip1/BCL11A (Abcam, ab19487); anti-c-Rel (Cell Signaling Technology, no. 4727); and anti- $\beta$-actin (Sigma-Aldrich, A2066). An ECL kit (Amersham Pharmacia Biotech Inc.) was used to develop the membranes, and the resultant films were analyzed using a UV densitometry machine (GeneSnap and Gene Tools software). The absorbance values for the tested proteins were normalized to the corresponding $\beta$-actin absorbance values, and the average normalized values for the proteins from 3 independent biological replicates for subjects and controls were used to generate 
graphs with protein expression comparison between cases and controls. For USP34 expression analysis, urea-based WCEs were prepared: $9 \mathrm{M}$ urea, $50 \mathrm{mM}$ Tris-HCL, $\mathrm{pH} \mathrm{7.5,} \mathrm{and} 10 \mathrm{mM}$ 2-mercaptoethanol with sonication (15 seconds at 30\% amplitude using a micro-tip; Sigma-Aldrich). WCEs were blotted using anti-USP34 Ab (Bethyl, A300-824).

Indirect immunofluorescence. XPO1 and USP34 function in LCLs was determined using anti-rpS5 (Santa Cruz Biotechnology Inc., C-20 sc-169174) and anti-53BP1 (Bethyl, A300-272A), respectively. rpS5 is one of the XPO1 cargos (37), and 53BP1 is a marker of DNA double-strand repair efficiency, a process proposed to be regulated by USP34 (26). Images were captured using Simple PCI software on the Zeiss Axioplan platform. For rpS5 nuclear export analysis, LCLs were also treated with leptomycin B, an XPO1 inhibitor (Sigma-Aldrich, $87081-35-4)$ at $20 \mathrm{ng} / \mathrm{ml}$ for 3 hours. For IR-induced 53BP1 foci formation, LCLs were irradiated with 3 Gy.

Immunohistochemical staining of brain tissues. Protein expression for the 4 genes (XPO1, USP34, BCL11A, and $R E L$ ) was determined using The Human Protein Atlas database (http://proteinatlas.org). In addition, detailed immunohistochemical analysis was performed on formalin-fixed wax sections of brain from a fetus (intrauterine death, with abnormal kidney and gonads) and from a 4-year-old girl who died from lymphocytic thyroiditis, with no evidence of brain involvement in either of the 2 cases. Anti-XPO1 and anti-USP34 Abs were used according to standard protocols. Images were captured and analyzed for staining patterns by a certified neuropathologist (CD).

Functional enrichment analysis. The WEB-based GEne SeT AnaLysis Toolkit (WebGestalt, http://bioinfo.vanderbilt.edu/webgestalt/) was used for functional enrichment analysis of genes from the $2 \mathrm{p} 15 \mathrm{p} 16.1$ region. The genes deleted in $50 \%$ of the patients with a $2 \mathrm{p} 15 \mathrm{p} 16.1$ microdeletion region were uploaded on WebGestalt. Subsequent enrichment analysis was based on the 16 unique Entrez Gene IDs, 15 of which were recognized by WebGestalt. Parameters used for all enrichment analysis modules included the commonly used reference gene sets (all genes in a genome), the multiple test adjustment default method proposed by Benjamini and Hochberg (59). WebGestalt uses Pathway Commons API to search pathway names in the Pathway Commons web source (http://www.pathwaycommons.org) to obtain the detailed information for the searched pathway. The top-10 pathways using hypergeometric statistics and with a minimum of 2 genes criteria were used.

$N F-\kappa B$ pathway analysis. Because NF- $\kappa \mathrm{B}$ was the top enriched pathway, we tested its signaling capacity in patients' LCLs. Abs from the NF-кB Pathway Sampler kit (Cell Signaling Technology, no. 9936) were used following treatment of LCLs with PMA as indicated (Supplemental Figure 4). Cytoplasmic extracts were prepared by treating cell pellets for 1 hour on ice in hypotonic detergent lysis buffer containing $50 \mathrm{mM}$ Tris- $\mathrm{HCl}$, pH 7.5, 150 mM NaCl, 2 mM EDTA, 2 mM EGTA, 25 mM NaF, 25 mM $\beta$-glycerolphosphate, $0.1 \mathrm{mM}$ Na-orthovanadate, $0.2 \%$ Triton X-100, 0.3\% octylphenoxypolyethoxyethanol (IGEPAL), and protease inhibitor cocktail tablets (Roche, no. 04693116001). Nuclei were pelleted by centrifugation, and protein concentration in the supernatant was quantified by Bradford Assay. Nuclear extract was prepared by treating the nuclear pellet with urea-based WCE buffer as described above.

In vivo zebrafish-knockdown analysis. Zebrafish orthologs of human 2p15p16.1 genes and their genomic arrangements (synteny) were identified using Ensembl, The Zebrafish Model Organism Database (ZFIN. org) and the UCSC genome browser, with sequence alignment and comparisons. The zebrafish protein sequences and functional domains were extracted from Ensembl and compared with the human counterparts by the Clustal Omega Multiple Sequence Alignment tool (EMBL-EBI).

Zebrafish (Danio rerio) WT AB embryos (60) were raised and maintained as previously described (61). Genespecific 25-base morpholino oligomers (MO) were designed by Gene Tools, LLC, to bind/block the splice donor or acceptor site (SB-MO), closest to the 5' end of the expected primary RNA. In cases in which a SB-MO failed to knockdown the gene, another MO was designed to target the genes mRNA translation initiation site (TBMO). The designed MO sequences are listed in Supplemental Table 8. As an experimental control for each gene, a gene-mismatch control MO (MM), representing a scrambled gene sequence, was also designed and injected into age- and clutch-matched zebrafish embryos as the gene MO. All MO were modified at the 3' end with carboxyfluorescein to visualize location and equal distribution of the MO solution in the post-injection embryos.

For injections, 1- to 2-cell-stage embryos were obtained from natural spawning of WT zebrafish. Approximately, 5 nanoliters of increasing concentrations ( $5 \mathrm{ng}, 7.5 \mathrm{ng}$, and $10 \mathrm{ng}$ ) of gene MO were injected into the yolk sac of the embryos with $0.1 \%$ phenol red using a variable INJECT+MATIC microinjector. The morphant embryos were grown at $28.5^{\circ} \mathrm{C}$ and observed for morphological changes under a stereoscopic microscope and fixed in $4 \%$ paraformaldehyde at specific developmental time points. A p53 MO was 
used to suppress off-target MO-induced apoptosis (62). Typically, $4 \mathrm{ng} \mathrm{MO}$ was coinjected with 1.5-fold p53 MO (i.e., $6 \mathrm{ng}$ ) to assess whether the phenotype was genuine.

Morphant embryos were assessed visually for phenotypic defects by live imaging at 1 and 3 dpf. Ventral and lateral images of 3-dpf fish were taken from 50 embryos per injection at the same magnification ( $\times 115$ and $\times 50$ for head/brain and whole-body images, respectively) using a Leica MZ16F stereomicroscope. Using ImageJ software (NIH), the distance between the eyes, the size of the otic vesicles, and the trunk length of 5 somites were measured as surrogate assays for microcephaly assessment, ear size, and body growth in human subjects, respectively; as these features are recurrent in patients with 2p15p16.1 microdeletion. The ratio of MM control and MO-injected embryos' head, otic vesicle, and somitic length size measurements $3 \mathrm{dpf}$ (normalized ratio) was plotted and presented as the mean $\pm \mathrm{SD}$. The phenotype assessment was performed in at least 3 independent injection experiments.

The embryos were treated with $200 \mu \mathrm{M} N$-phenylthiourea (PTU; Sigma-Aldrich, catalog P7629) 24 hours post fertilization (hpf) to inhibit melanogenesis for better visibility and assessment of the developing internal organs (e.g., brain) (63). Brain structures were evaluated $1 \mathrm{dpf}$ by analyzing the forebrain, midbrain, and hindbrain morphology compared with control brains. Brains that deviated from a normal appearance were recorded and their percentage presented for MO fish compared with controls.

Gene-knockdown confirmation analysis. Each MO knockdown was confirmed the RNA or protein level, depending on the nature of the MO used, by RT-PCR (for SB-MO) or Western blotting (for TB-MO). The detailed explanation of the RT-PCR assay used to confirm SB-MO knockdown was previously published (64). Total RNA was extracted from thirty 1- to 3-dpf gene MO or MM control-injected embryos. Forward and reverse primers spanning the SB-MO-binding site, listed on Supplemental Table 9, were then designed and used to amplify the region of the SB-MO-mediated gene splice blockage by PCR using Taq polymerase (Invitrogen, no. 18038042). The amplified PCR products were then separated on a 3\% agarose gel, and the presence of a truncated band and/or a diminished normal-sized band in the sample PCR product compared with control indicated successful splice blockage and gene knockdown. For 1 gene, rel, the knockdown was not achieved with 2 independently designed SB-MO (as was confirmed with RT-PCR and gel electrophoresis); therefore, we used a TB-MO, which resulted in successful knockdown as confirmed by extraction of total protein from thirty 3-dpf rel-MO-injected and control embryos (using radioimmunoprecipitation assay [RIPA] lysis and extraction buffer from Thermo Scientific, no. 89900) and Western blotting using rabbit polyclonal anti-Rel (AnaSpec, no. 55483) and infrared fluorescent dye (IRDye 680 LT-tagged goat anti-rabbit secondary Ab; LI-COR Biosciences, no. 926-68021). The membrane was scanned and the protein band visualized with a LI-COR Odyssey infrared imager.

Statistics. All pairwise comparisons were performed using the 2-tailed $t$ test unless otherwise stated. For human protein measurement analyses, differences were considered highly significant if the $P$ value was less than 0.01 , while not significant if the $P$ value was greater than 0.05 . For zebrafish measurements, differences were considered highly significant if the $P$ value was less than 0.0001 , significant if the $P$ value was less than 0.001 , and not significant if the $P$ value was greater than 0.01 .

Study approval. Recruitment of subjects was performed with the approval of the UBC Clinical Research Ethics Committee. Participants were enrolled in the study after providing informed consent and were clinically assessed by their respective physicians. Written consent was obtained for publication of the patients' images shown in Figure 1. Zebrafish (Danio rerio) WT AB embryos (60) were raised and maintained as previously described (61) and with the approval of the IACUC of the UBC and in accordance with the Canadian Council of Animal Care.

\section{Author contributions}

$\mathrm{HB}$ and $\mathrm{CB}$ conducted experiments, acquired and analyzed data, and wrote the manuscript. YQ analyzed data. RC, IA, DA, JW, SM, and XS conducted experiments and acquired and analyzed data. CD analyzed brain immunohistochemical data. BM, RSW, MJMN, JE, AL, and SMEL recruited patients and reviewed the manuscript. MO'D and CYGE supervised data acquisition and analysis and reviewed the manuscript. ERS coordinated efforts and contributed to writing and review of the manuscript.

\section{Acknowledgments}

The authors appreciate the collaboration and support of the participating patients and their families. $\mathrm{H}$. Bagheri is recipient of Child and Family Research Institute (CFRI) graduate studentship and E. Rajcan- 
Separovic and S.M.E. Lewis are recipients of Michael Smith Foundation of Health Research career salary awards. Canadian Institutes of Health Research operational funding was awarded to E. Rajcan-Separovic (MOP 74502). R. Colnaghi, D. Alcantara, I. Abramowicz, and M. O’Driscoll are supported by a Cancer Research UK (CR-UK) program award given to M. O'Driscoll (C24110/A15394). C.Y. Gregory-Evans is a recipient of a Sharon Stewart Trust Grant 2014. H.Bagheri is the recipient of the Child and Family Research Institute graduate award. DNA from the subject in the study by Chabchoub et al. was provided courtesy of Thomy DeRavel. The authors wish to thank Wendy Robinson's laboratory for access to the ABI PRISM 310 Genetic Analyzer.

Address correspondence to: Mark O’Driscoll, Human DNA Damage Response Disorders Group, Genome Damage and Stability Centre, School of Life Sciences, University of Sussex, Falmer, Brighton, BN1 9RQ, United Kingdom. Phone: 44.0.1273.877-515; E-mail: m.o-driscoll@sussex.ac.uk. Or to: Cheryl Y. Gregory-Evans, Department of Ophthalmology and Visual Sciences, University of British Columbia, Eye Care Centre, 2550 Willow Street, Vancouver, BC, V5Z 3N9, Canada. Phone: 604.875.5529; E-mail: cge30@ mail.ubc.ca. Or to: Evica Rajcan-Separovic, Department of Pathology and Laboratory Medicine, University of British Columbia, Child and Family Research Institute, 950 West $28^{\text {th }}$, Room 309, Vancouver, BC, V5Z 4H4, Canada. Phone: 604.875.3121; E-mail: eseparovic@cw.bc.ca.

Robert S. Wildin's present address is: National Human Genome Research Institute, Bethesda, Maryland, USA.

1. Rajcan-Separovic E, et al. Clinical and molecular cytogenetic characterisation of a newly recognised microdeletion syndrome involving 2p15-16.1. J Med Genet. 2007;44(4):269-276.

2. Shimojima K, Okamoto N, Yamamoto T. Characteristics of 2p15-p16. 1 microdeletion syndrome: Review and description of two additional patients. Congenit Anom (Kyoto). 2015;55(3):125-132.

3. Balci TB, Sawyer SL, Davila J, Humphreys P, Dyment DA. Brain malformations in a patient with deletion 2p16. 1: A refinement of the phenotype to BCL11A. Eur J Med Genet. 2015;58(6-7):351-354.

4. Basak A, et al. BCL11A deletions result in fetal hemoglobin persistence and neurodevelopmental alterations. J Clin Invest. 2015;125(6):2363-2368.

5. Ronzoni L, Saletti V, Scuvera G, Esposito S, Milani D. Response to "Characteristics of 2p15-p16.1 microdeletion syndrome: Review and description of two additional patients". Congenit Anom (Kyoto). 2015;55(4):191-192.

6. Ottolini KM, Turner CE, Gada SM. Hypogammaglobulinemia and impaired antibody response in a child with chromosome 2p15-16.1 microdeletion syndrome. Ann Allergy Asthma Immunol. 2015;115(2):153-155.

7. Florisson JM, Mathijssen IM, Dumee B, et al. Complex craniosynostosis is associated with the 2p15p16.1 microdeletion syndrome. Am J Med Genet A. 2013;161A(2):244-253.

8. Peter B, Matsushita M, Oda K, Raskind W. De novo microdeletion of BCL11A is associated with severe speech sound disorder Am J Med Genet A. 2014;164A(8):2091-2096.

9. Hancarova M, Simandlova M, Drabova J, Mannik K, Kurg A, Sedlacek Z. A patient with de novo 0.45 Mb deletion of 2p16. 1: the role of BCL11A, PAPOLG, REL, and FLJ16341 in the 2p15-p16.1 microdeletion syndrome. Am J Med Genet A. 2013;161A(4):865-870.

10. Fannemel M, et al. Haploinsufficiency of XPO1 and USP34 by a de novo $230 \mathrm{~kb}$ deletion in $2 \mathrm{p} 15$, in a patient with mild intellectual disability and cranio-facial dysmorphisms. Eur J Med Genet. 2014;57(9):513-519.

11. Prontera P, et al. Deletion 2p15-16.1 syndrome: case report and review. Am J Med Genet A. 2011;155A(10):2473-2478.

12. De Leeuw N, et al. A newly recognised microdeletion syndrome involving 2p15p16. 1: narrowing down the critical region by adding another patient detected by genome wide tiling path array comparative genomic hybridisation analysis. JMed Genet. 2008;45(2):122-124.

13. Félix TM, Petrin AL, Sanseverino MTV, Murray JC. Further characterization of microdeletion syndrome involving 2p15-p16. 1. Am J Med Genet A. 2010;152A(10):2604-2608.

14. Liang JS, et al. A newly recognised microdeletion syndrome of 2p15-16.1 manifesting moderate developmental delay, autistic behaviour, short stature, microcephaly, and dysmorphic features: a new patient with 3.2 Mb deletion. JMed Genet. 2009;46(9):645-647.

15. Jorgez CJ, et al. Genitourinary defects associated with genomic deletions in 2p15 encompassing OTX1. PLoS One. 2014;9(9):e107028.

16. Piccione M, et al. Interstitial deletion of chromosome 2p15-16.1: Report of two patients and critical review of current genotypephenotype correlation. Eur J Med Genet. 2012;55(4):238-244.

17. Hucthagowder V, et al. Chromosome 2p15p16. 1 microdeletion syndrome: $2.5 \mathrm{Mb}$ deletion in a patient with renal anomalies, intractable seizures and a choledochal cyst. Eur J Med Genet. 2012;55(8):485-489.

18. Chabchoub E, Vermeesch JR, de Ravel T, de Cock P, Fryns JP. The facial dysmorphy in the newly recognised microdeletion 2p15-p16. 1 refined to a $570 \mathrm{~kb}$ region in 2p15. J Med Genet. 2008;45(3):189-192.

19. Huang N, Lee I, Marcotte EM, Hurles ME. Characterising and predicting haploinsufficiency in the human genome. PLoS Genet. 2010;6(10):e1001154.

20. Honda T, Nakajima K. Mouse Disabled1 (DAB1) is a nucleocytoplasmic shuttling protein. J Biol Chem. 2006;281(50):3895138965. 
21. Simon-Areces J, Acaz-Fonseca E, Ruiz-Palmero I, Garcia-Segura L-M, Arevalo M-A. A CRM1-mediated nuclear export signal is essential for cytoplasmic localization of neurogenin 3 in neurons. PLoS One. 2013;8(1):e55237.

22. Sarnico I, et al. NF-кB dimers in the regulation of neuronal survival. Int Rev Neurobiol. 2009;85:351-362.

23. Salles A, Romano A, Freudenthal R. Synaptic NF-кB pathway in neuronal plasticity and memory. J Physiol Paris. 2014;108(46):256-262.

24. Poalas K, et al. Negative regulation of NF-кB signaling in T lymphocytes by the ubiquitin-specific protease USP34. Cell Commun Signal. 2013;11(1):25.

25. Lui TT, et al. The ubiquitin specific protease USP34 regulates axin stability and Wnt/ $\beta$-catenin signaling. Mol Cell Biol. 2011;31(10):2053-2065.

26. Sy SM, et al. The ubiquitin specific protease USP34 promotes ubiquitin signaling at DNA double-strand breaks. Nucleic Acids Res. 2013;41(18):8572-8580

27. Huang HT, Chen SM, Pan LB, Yao J, Ma HT. Loss of function of SWI/SNF chromatin remodeling genes leads to genome instability of human lung cancer. Oncol Rep. 2015;33(1):283-291.

28. Wiegreffe C, et al. Bcl11a (Ctip1) controls migration of cortical projection neurons through regulation of Sema3c. Neuron. $2015 ; 87(2): 311-325$.

29. Golzio C, et al. KCTD13 is a major driver of mirrored neuroanatomical phenotypes of the $16 \mathrm{p} 11.2$ copy number variant. Nature. 2012;485(7398):363-367.

30. Dauber A, et al. SCRIB and PUF60 are primary drivers of the multisystemic phenotypes of the 8q24. 3 copy-number variant. Am J Hum Genet. 2013;93(5):798-811.

31. Carvalho CM, et al. Dosage changes of a segment at 17p13. 1 lead to intellectual disability and microcephaly as a result of complex genetic interaction of multiple genes. Am J Hum Genet. 2014;95(5):565-578.

32. García-Santisteban I, Bañuelos S, Rodríguez JA. A global survey of CRM1-dependent nuclear export sequences in the human deubiquitinase family. Biochem J. 2012;441(1):209-217.

33. Muller PA, et al. Nuclear-cytosolic transport of COMMD1 regulates NF-кB and HIF-1 activity. Traffic. 2009;10(5):514-527.

34. Walker CJ, et al. Preclinical and clinical efficacy of XPO1/CRM1 inhibition by the karyopherin inhibitor KPT-330 in Ph+ leukemias. Blood. 2013;122(17):3034-3044.

35. Funnell AP, et al. 2p15-p16.1 microdeletions encompassing and proximal to BCL11A are associated with elevated HbF in addition to neurologic impairment. Blood. 2015;126(1):89-93.

36. Gutzman JH, Sive H. Epithelial relaxation mediated by the myosin phosphatase regulator Mypt1 is required for brain ventricle lumen expansion and hindbrain morphogenesis. Development. 2010;137(5):795-804.

37. Thomas F, Kutay U. Biogenesis and nuclear export of ribosomal subunits in higher eukaryotes depend on the CRM1 export pathway. J Cell Sci. 2003;116(12):2409-2419.

38. Tabe Y, et al. Ribosomal biogenesis and translational flux inhibition by the Selective Inhibitor of Nuclear Export (SINE) XPO1 antagonist KPT-185. PLoS One. 2015;10(9):e0137210.

39. Forbes DJ, Travesa A, Nord MS, Bernis C. Nuclear transport factors: global regulation of mitosis. Curr Opin Cell Biol. 2015;35:78-90.

40. Liu X, et al. 2p15-p16.1 microdeletion syndrome: molecular characterization and association of the OTX1 and XPO1 genes with autism spectrum disorders. Eur J Hum Genet. 2011;19(12):1264-1270.

41. Puente XS, et al. Whole-genome sequencing identifies recurrent mutations in chronic lymphocytic leukaemia. Nature. 2011;475(7354):101-105.

42. Haines JD, et al. Nuclear export inhibitors avert progression in preclinical models of inflammatory demyelination. Nat Neurosci. 2015;18(4):511-520.

43. Bauer DE, Orkin SH. Hemoglobin switching's surprise: the versatile transcription factor BCL11A is a master repressor of fetal hemoglobin. Curr Opin Genet Dev. 2015;33:62-70.

44. Lanzillotta A, et al. NF-кB in innate neuroprotection and age-related neurodegenerative diseases. Front Neurol. $2015 ; 6: 98$.

45. Sarnico I, et al. NF- $\mathrm{B}$ p50/RelA and c-Rel-containing dimers: opposite regulators of neuron vulnerability to ischaemia. $J$ Neurochem. 2009;108(2):475-485.

46. van de Sluis B, et al. Increased activity of hypoxia-inducible factor 1 is associated with early embryonic lethality in Commd1 null mice. Mol Cell Biol. 2007;27(11):4142-4156.

47. Maxwell M, et al. Pex13 inactivation in the mouse disrupts peroxisome biogenesis and leads to a Zellweger syndrome phenotype. Mol Cell Biol. 2003;23(16):5947-5957.

48. Lee MJ, et al. Hereditary sensory neuropathy is caused by a mutation in the $\Delta$ subunit of the cytosolic chaperonin-containing t-complex peptide-1 (Cct4) gene. Hum Mol Genet. 2003;12(15):1917-1925.

49. Engel E, Viargues P, Mortier M, et al. Identifying USPs regulating immune signals in Drosophila: USP2 deubiquitinates Imd and promotes its degradation by interacting with the proteasome. Cell Commun Signal. 2014;12(1):41.

50. Ersing I, Bernhardt K, Gewurz BE. NF-кB and IRF7 pathway activation by Epstein-Barr virus Latent Membrane Protein 1. Viruses. 2013;5(6):1587-1606.

51. Visel A, Minovitsky S, Dubchak I, Pennacchio LA. VISTA Enhancer Browser - a database of tissue-specific human enhancers Nucleic Acids Res. 2007;35(suppl 1):D88-D92.

52. Mimouni-Bloch A, Yeshaya J, Kahana S, Maya I, Basel-Vanagaite L. A de-novo interstitial microduplication involving 2p16.1p15 and mirroring 2p16.1-p15 microdeletion syndrome: Clinical and molecular analysis. Eur J Paediatr Neurol. 2015;S10903798(6):141-145.

53. Kwiecinska A, et al. Amplification of $2 \mathrm{p}$ as a genomic marker for transformation in lymphoma. Genes Chromosomes Cancer. 2014;53(9):750-768

54. Rinaldi A, et al. Single nucleotide polymorphism-arrays provide new insights in the pathogenesis of post-transplant diffuse large B-cell lymphoma. Br J Haematol. 2010;149(4):569-577.

55. Luo WJ, et al. Epstein-Barr virus is integrated between REL and BCL-11A in American Burkitt lymphoma cell line (NAB-2). Lab Invest. 2004;84(9):1193-1199. 
56. Qiao Y, et al. Clinical application of 2.7 M Cytogenetics array for CNV detection in subjects with idiopathic autism and/or intellectual disability. Clin Genet. 2013;83(2):145-154

57. Charbonnier F, et al. Detection of exon deletions and duplications of the mismatch repair genes in hereditary nonpolyposis colorectal cancer families using multiplex polymerase chain reaction of short fluorescent fragments. Cancer Res. 2000;60(11):2760-2763

58. Harvard C, et al. Understanding the impact of 1q21.1 copy number variant. Orphanet J Rare Dis. 2011;6:54.

59. Benjamini Y, Hochberg Y. Controlling the false discovery rate: a practical and powerful approach to multiple testing. $J R$ Stat Soc Series B Methodol. 1995;57(1):289-300.

60. Johnson SL, Midson CN, Ballinger EW, Postlethwait JH. Identification of RAPD primers that reveal extensive polymorphisms between laboratory strains of zebrafish. Genomics. 1994;19(1):152-156.

61. Viringipurampeer IA, et al. Pax2 regulates a fadd-dependent molecular switch that drives tissue fusion during eye development. Hum Mol Genet. 2012;21(10):2357-2369.

62. Robu ME, et al. p53 activation by knockdown technologies. PLoS Genet. 2007;3(5):e78.

63. Karlsson J, von Hofsten J, Olsson PE. Generating transparent zebrafish: a refined method to improve detection of gene expression during embryonic development. Mar Biotechnol (NY). 2001;3(6):522-527.

64. Morcos PA. Achieving targeted and quantifiable alteration of mRNA splicing with Morpholino oligos. Biochem Biophys Res Commun. 2007;358(2):521-527. 